\title{
Multi-View Clustering via Multi-Manifold Regularized Non-negative Matrix Factorization
}

\author{
Linlin Zong, Xianchao Zhang*, Long Zhao, Hong Yu, Qianli Zhao \\ Dalian University of Technology, Dalian, China, 116620
}

\begin{abstract}
Non-negative matrix factorization based multi-view clustering algorithms have shown their competitiveness among different multi-view clustering algorithms. However, non-negative matrix factorization fails to preserve the locally geometrical structure of the data space. In this paper, we propose a multi-manifold regularized non-negative matrix factorization framework (MMNMF) which can preserve the locally geometrical structure of the manifolds for multi-view clustering. MMNMF incorporates consensus manifold and consensus coefficient matrix with multi-manifold regularization to preserve the locally geometrical structure of the multi-view data space. We use two methods to construct the consensus manifold and two methods to find the consensus coefficient matrix, which leads to four instances of the framework. Experimental results show that the proposed algorithms outperform existing non-negative matrix factorization based algorithms for multi-view clustering.
\end{abstract}

Keywords: Non-negative matrix factorization, Multi-view clustering, Multi-manifold, Locally Linear Embedding (LLE)

\footnotetext{
${ }^{\star}$ A preliminary version of this paper was presented in the 14th IEEE International Conference on Data Mining (ICDM-14) Zhang et al. (2014).

${ }^{*}$ Corresponding author. Tel.:+86-411-84709360; fax:+86-411-84709360

Email addresses: zonglinlin324@gmail.com (Linlin Zong), xczhang@dlut.edu.cn (Xianchao Zhang*), zhaolong.324@163.com (Long Zhao), hongyu@dlut.edu.cn (Hong Yu), sconan32@live.com (Qianli Zhao)
}

Preprint submitted to Journal of ${ }^{A} T_{E} X$ Templates

November 28, 2016

(C) 2017. This manuscript version is made available under the Elsevier user license http://www.elsevier.com/open-access/userlicense/1.0/ 


\section{Introduction}

Clustering, which aims to partition a set of data into groups according to the data points' similarities, is a fundamental topic in machine learning and data mining. Many real-world datasets are comprised of different views, and the 5 views often provide compatible and complementary information. Thus extracting information from multiple views gains better clustering performance than from a single view. Multi-view clustering (Bickel \& Scheffer (2004)) has become a hot topic since the past decade and many algorithms have been proposed.

Also since the past decade, there has been some significant developments Aggarwal \& Reddy (2013)) in the use of non-negative matrix factorization (NMF) for clustering. In particular, NMF is shown to be equivalent to relaxed $k$-means (Ding et al. (2005)), and symmetric NMF is closely related to spectral clustering (Kuang et al. (2012)). Recently, several NMF based multi-view clustering algorithms have been proposed (Akata et al. (2011); Liu et al. (2013);

15 Singh \& Gordon (2008)). These algorithms learned the underlying clustering structure embedded in multiple views efficiently as well as kept different coefficient matrices comparable and meaningful. However, NMF cannot preserve the locally geometrical structure of the data space (Cai et al. (2011)), which is essential for the algorithms to find the true cluster structures. For single view datasets, Cai et al. (2011) proposed the graph regularized NMF (GNMF) which imposed manifold regularization on NMF formulation to preserve the locally geometrical structure. Nevertheless, as we will show later in the experimental section, GNMF does not achieve very good performance if it is directly used as a variation of multi-view $\mathrm{NMF}$.

In this paper, we propose a multi-manifold regularized non-negative matrix factorization framework (MMNMF) which can preserve the locally geometrical structure of multiple views. MMNMF incorporates consensus manifold and consensus coefficient matrix with multi-manifold regularization to preserve the locally geometrical structure of the multi-view data space. The framework is 
the consensus manifold lead to different realizations of the framework. In this paper, we use linear combination and multi-view locally linear embedding (LLE) to construct the consensus manifold, and use linear combination and consensus matrix approximation (Liu et al. (2013) ) to find the consensus coefficient matrix.

35 Finally, we get four instances of the framework. Experimental results show that the proposed algorithms outperform existing NMF based multi-view clustering algorithms.

A preliminary version of this paper was presented in the 14th IEEE International Conference on Data Mining (ICDM-14) (Zhang et al. (2014)). In this paper, we extend the preliminary version from the following aspects. (1) We use Frobenius norm in the instances; (2) We add a new method, multi-view locally linear embedding (LLE), to construct the consensus manifold. Consequently, two more instances of the framework are generated; (3) We use EMDA (Beck \& Teboulle (2003)) to adjust the parameter $\alpha$ and $\mu$ automatically; (4)

${ }_{45}$ We provide the proof of the convergence of the algorithm; (5) We investigate parameter settings empirically; (6) We compare the running time of MMNMF with that of the baselines; (7) We report the running time of updating matrices and determining parameters separately.

\section{Related work}

\subsection{Multi-view clustering}

Many multi-view clustering algorithms have been proposed over the past few years, and they can be roughly classified into four categories. The first category of algorithms integrated the loss function of each single view and optimized the new objective function (Bickel \& Scheffer (2004); Cai et al. (2013)). The second category of algorithms looked for the best graph adjacency matrix firstly, then used spectral graph theory or other graph based techniques to solve the problem (Kumar et al. (2011); Kumar \& Daumé (2011); Zhou \& Burges (2007)). The third category of algorithms constructed graph for every view firstly, then developed a unified graph and used graph clustering methods (Greene \& Cunningham 
(2013)). The fourth category of algorithms were based on NMF, various constraints have been applied to the coefficient matrix to cluster the data points (Akata et al. (2011); Liu et al. (2013); Singh \& Gordon (2008)).

The spectral clustering based methods and NMF based methods have received increasing attention. In spectral clustering based methods, the key point is to obtain an adjacency matrix. Kumar et al. (Kumar et al. (2011); Kumar \& Daumé (2011) ) adopted co-training techniques to get the adjacency matrix iteratively. Zhou \& Burges (2007) combined multiple regularized adjacency matrix linearly. For NMF based methods, the key point is to get a coefficient matrix with low rank. Some methods (Akata et al. (2011); Singh \& Gordon (2008)) decomposed the original matrices into discriminative basis matrices and a shared coefficient matrix by using correlational analysis. Liu et al. (2013) decomposed the original matrices into discriminative basis matrices and discriminative coefficient matrices, and found a consensus coefficient matrix to equilibrate all the coefficient matrices. Li et al. (2014) clustered partially mapped multi-view data

75 by distinguishing coefficient vectors of the mapped data points from coefficient vectors of the individual data points. Zhang et al. (2015) utilized inter-view constraints to deal with unmapped multi-view data.

In addition, Greene \& Cunningham (2013) constructed a unified graph from multiple social networks. Graph combination is also a new direction for multiview clustering.

\subsection{NMF and manifold regularization}

․ NMF is a dimension reduction technique for non-negative data Lee \& Seung (1999, 2001)). Denote $X=\left[x_{1}, x_{2}, \ldots, x_{n}\right] \in R_{+}^{m \times n}$ as the original $m$ dimensional data. NMF aims to find two low rank factor matrices $U \in R_{+}^{m \times r}$

85 and $V \in R_{+}^{n \times r}$ whose product is a good approximation to $X, X \approx U V^{T}$, where $r$ is the low rank and $r \ll \min (m, n)$. On account of learning compact representation, $U$ can be interpreted as basis matrix and $V$ can be interpreted as coefficient matrix. To obtain a good approximation of $X$, we need to evaluate the reconstruction error between $X$ and $U V^{T}$, one commonly used measure is 
Frobenius norm,

$$
D_{F}\left(X \| U V^{T}\right)=\left\|X-U V^{T}\right\|_{F}^{2}
$$

where $\|\cdot\|_{F}$ denotes Frobenius norm.

NMF has been widely used in clustering because of its compact learning ability. If $r$ represents the number of clusters, $\mathbf{V}$ shows the cluster indicator information. However, NMF fails to discover the intrinsic geometry of the data space, which is essential to the real applications. To preserve the locally geometrical structure of the data space, Cai et al. (2011) imposed graph regularization on NMF (GNMF). The method is based on manifold assumption which means that if two data points $x_{i}$ and $x_{j}$ are close in the intrinsic geometry of the data distribution, then the representations of the two points in the new basis are also close to each other. In Cai et al. (2011), graph Laplacian (Chung (1997)) was used to discretely approximate to the manifold and $J_{\text {manifold }}$ was used to measure the smoothness of the mapping function along the geodesics in the intrinsic geometry of the dataset.

$$
\left.J_{\text {manifold }}=\sum_{i, j=1}^{n}\left(\left\|V_{i .}-V_{j .}\right\|^{2} A_{i j}\right)\right)=\operatorname{tr}\left(V^{T} L V\right),
$$

where $\operatorname{tr}(\cdot)$ denotes the trace of a matrix, $V_{i}$. is the $i$-th row of $V, L$ is the Laplacian matrix and $L=D-A, D$ is a diagonal matrix and $D_{i i}=\sum_{j} A_{i j}$, $A=\left\{A_{i j}\right\}_{i, j=1}^{n}$ denotes the adjacency matrix of the graph.

95 口 In addition, multi-manifold is widely studied in single-view clustering. Liu et al. 2005) used sparse variant of NMF for multi-manifold learning. Li et al. (2013) considered the case when data points reside on overlapped manifolds. Shen \& Si (2010) assumed that data points are drawn from multiple manifolds, and they mined intrinsic geometry on individual manifold. Wang et al. (2011) proposed a local and structural consistency criterion for hybrid manifold clustering.

Taking the previous contributions into account, our framework is the first one that incorporates multi-manifold regularization with NMF based multi-view 
clustering.

\subsection{Locally linear embedding}

Locally linear embedding (LLE) (Roweis \& Saul (2000)) has been widely used in learning similarity matrix. Some methods (Roweis \& Saul (2000); Wang \& Zhang (2008)) previously fixed the number of neighbors and used l2-norm in objective function. The others (Yang et al. (2010); Cheng et al. (2009); Yan

10 \& Wang (2009)) minimized the $l 1$-norm and learned a sparse adjacency matrix without fixed neighborhood for each point. In addition, Tian \& Kuang (2012) tried to utilize the global linear neighbors and finally turned the problem into non-negative matrix factorization.

Supposing that data points $X$ are sampled from some underlying manifolds, based on simple geometric intuitions, each data point and its neighbors are expected to lie on or close to a locally linear patch of the manifold Roweis \& Saul (2000)). The local geometry of these patches can be characterized by reconstructing each data point with its neighbors, shown as Eq. (3) (Wang \& Zhang (2008)),

$$
\begin{aligned}
\epsilon(W)= & \sum_{i}\left\|x_{i}-\sum_{j: x_{j} \in N\left(x_{i}\right)} W_{i j} x_{j}\right\|^{2} \\
\text { s.t. } & \sum_{j: x_{j} \in N\left(x_{i}\right)} W_{i j}=1,
\end{aligned}
$$

where $N\left(x_{i}\right)$ is the set of neighbors of $x_{i}$ and $W_{i j}=0$ if $x_{j} \notin N\left(x_{i}\right)$. W is asymmetric. We set the adjacency matrix $A=\left(W+W^{T}\right) / 2$ when we use $W$ in manifold regularization.

\section{Multiple manifold regularized non-negative matrix factorization}

\subsection{Overview of the framework}

Let $X^{(1)}, X^{(2)}, \ldots, X^{\left(n_{v}\right)}$ be the data of all the views and $X^{(v)} \in R_{+}^{m^{(v)} \times n}, v=$ $1,2, \ldots, n_{v}, m^{(v)}$ be the dimension of $v$-th view, $n$ be the number of data points and $n_{v}$ be the number of views. Denote $A^{(v)}=\left\{A_{i j}^{(v)}\right\}_{i, j=1}^{n}$ as the adjacency 
matrix of $v$-th view, $D^{(v)}$ as a diagonal matrix and $D_{i i}^{(v)}=\sum_{j} A_{i j}^{(v)}, L^{(v)}=$ $D^{(v)}-A^{(v)}$ as the Laplacian matrix of $v$-th view.

Manifold assumption shows that the data points and their neighbors in each view lie on or near to a local patch of a manifold (discretely approximated by $\left.L^{(v)}\right)$. Naturally, multi-view datasets lie on or near to a local patch of multiple manifolds. Here, we introduce a consensus manifold $L^{*}$ as the balance of $L^{(v)}$ and a consensus coefficient matrix $V^{*}$ as the balance of $V^{(v)}$.

Let $D\left(V^{*} \| V^{(v)}\right)$ and $D\left(L^{*} \| L^{(v)}\right)$ represent cost functions by using $V^{(v)}$ to find $V^{*}$ and using $L^{(v)}$ to find $L^{*}$ respectively, then our multiple manifolds regularized non-negative matrix factorization framework (MMNMF) tries to minimize Eq. (4),

$$
\begin{aligned}
\Omega & =\sum_{v=1}^{n_{v}}\left(D\left(X^{(v)} \| U^{(v)} V^{(v) T}\right)+D\left(V^{*} \| V^{(v)}\right)+D\left(L^{*} \| L^{(v)}\right)\right) \\
& +\quad \lambda \operatorname{tr}\left(V^{* T} L^{*} V^{*}\right) \\
\text { s.t. } & \quad U^{(v)} \geq 0, V^{(v)} \geq 0, V^{*} \geq 0 .
\end{aligned}
$$

The first part of Eq. (4) is the deviation between the original matrix and the approximate matrix. In this paper, we use Frobenius norm (Eq. (1) to penalize it. The second part of Eq. (4) aims to minimize the difference between the consensus coefficient matrix and corresponding coefficient matrix in each view. The third part of Eq.4 aims to minimize the difference between the consensus manifold and corresponding manifold in each view. The fourth part of Eq. (4) ensures that each view satisfies the manifold assumption.

\subsection{Finding consensus manifold}

\subsubsection{Linear combination}

In this subsection, we find the consensus manifold by using the intrinsic manifold approximation. Supposing that the intrinsic manifold is embedded in a convex hull of all the manifolds (Geng et al. (2012); Li et al. (2013)), the intrinsic manifold can be seen as a linear approximation of the manifolds in all views, $L^{*}=\sum_{v} \mu_{v} L^{(v)}, \sum_{v} \mu_{v}=1, \mu_{v} \geq 0$. Here, we simply present the derivation 
from random walks on multiple undirected graphs. A random walk on a graph is a stochastic process which randomly jumps from vertex to vertex Aldous \& 155 Fill (2002)). For each view, we treat it as a graph $G^{(v)}=\left(V e, E^{(v)}, A^{(v)}\right)$, where $V e$ represents the set of vertices, $E^{(v)}$ indicates the edges between vertices, and $A^{(v)}$ shows the weights of edges.

Firstly, we consider random walk on each single graph. Denote $P^{(v)}$ as the transition probability and $\Pi^{(v)}$ as the stationary distribution, we can calculate $P^{(v)}$ and $\Pi^{(v)}$ according to the following formula (Von Luxburg (2007)):

$$
P_{i j}^{(v)}=\frac{A_{i j}^{(v)}}{D_{i i}^{(v)}} ; \quad \Pi_{i}^{(v)}=\frac{D_{i i}^{(v)}}{\operatorname{tr}\left(D^{(v)}\right)} .
$$

Nextly, for multiple graphs, we proceed a mixture of multiple random walks (Zhou \& Burges (2007)). Similarly, we define the transition probability $P^{*}$, and the stationary distribution $\Pi^{*}$ on mixture model, and we can calculate $\Pi^{*}$ according to the following formula:

$$
\Pi_{i}^{*}=\sum_{v} \mu_{v} \Pi_{i}^{(v)}=\sum_{v} \mu_{v} \frac{D_{i i}^{(v)}}{\operatorname{tr}\left(D^{(v)}\right)},
$$

where $\mu_{v} \geq 0$,and $\sum_{v} \mu_{v}=1, v=1,2, \ldots, n_{v}$. The constraints on $\mu_{v}$ show that each view should not have a negative contribution to the final result.

$$
\text { Let } \zeta_{i}^{(v)}=\mu_{v} \Pi_{i}^{(v)} / \sum_{j=1}^{n_{v}} \mu_{j} \Pi_{i}^{(j)} \text { Zhou \& Burges 2007), such that } \sum_{v} \zeta_{i}^{(v)}=
$$
1 and $\zeta_{i}^{(v)} \geq 0$, then we calculate $P^{*}$ as Eq. (7)

$$
P_{i j}^{*}=\sum_{v} \zeta_{i}^{(v)} P_{i j}^{(v)}
$$

It is easy to verify that $\Pi_{j}^{*}=\sum_{i} \Pi_{i}^{*} P_{i j}^{*}$. For mixture model, if $\operatorname{tr}\left(D^{(v)}\right)=C$ is a constant, we can get Eq. (8) which could result in $A^{*}=\sum_{v} \mu_{v} A^{(v)}$ and 


$$
\begin{aligned}
D^{*}=\sum_{v} \mu_{v} D^{(v)} . & \\
\frac{A_{i j}^{*}}{\operatorname{tr}\left(D^{*}\right)} & =P_{i j}^{*} \cdot \Pi_{i}^{*} \\
& =\sum_{v} \frac{\mu_{v} \Pi_{i}^{(v)}}{\sum_{j} \mu_{j} \Pi_{i}^{(j)}} \cdot \frac{A_{i j}^{(v)}}{D_{i i}^{(v)}} \cdot \sum_{j} \mu_{j} \Pi_{i}^{(j)} \\
& =\sum_{v} \mu_{v} \Pi_{i}^{(v)} \frac{A_{i j}^{(v)}}{D_{i i}^{(v)}} \\
& =\sum_{v} \mu_{v} \frac{A_{i j}^{(v)}}{\operatorname{tr}\left(D^{(v)}\right)} \\
& =\frac{\sum_{v} \mu_{v} A_{i j}^{(v)}}{\operatorname{tr}\left(\sum_{v} \mu_{v} D^{(v)}\right)} .
\end{aligned}
$$

So for multiple graphs, the weights of edges can be treated as a linear combination of normalized edge weights of single graph (the sum of all edge weights is a constant). Notice that normalization is necessary because weights on different graphs may be measured in very different scales.

Then, we can calculate the consensus Laplacian matrix $L^{*}$ as Eq. 9.

$$
L^{*}=D^{*}-A^{*}=\sum_{v} \mu_{v} L^{(v)}, \text { s.t. } \sum_{v} \mu_{v}=1, \mu_{v} \geq 0 .
$$

\subsubsection{Multi-view locally linear embedding}

In this subsection, we find the consensus manifold by using the LLE. Data points with multiple views can be reconstructed with their neighbors in each view. The distributions of different views may be different, thus the neighbors of one data point may vary in different views. To find the consensus manifold combining local geometry in different views, we reconstruct a data point with its neighbors in different views. Defining $I\left(x_{i}^{(v)}\right)$ as indexes of neighbors of $x_{i}^{(v)}$ in $v$-th view and $I\left(x_{i}\right)=\bigcup_{v} I\left(x_{i}^{(v)}\right)$ as indexes of neighbors of $x_{i}$ in all views, 
we try to find $W$ by using Eq. 10 ,

$$
\begin{aligned}
& \epsilon_{i}=\sum_{v} \rho_{v}\left\|x_{i}^{(v)}-\sum_{j \in I\left(x_{i}\right)} W_{i j} x_{j}^{(v)}\right\|^{2}+\|\rho\|^{2} \\
&=\sum_{v} \rho_{v}\left\|\sum_{j \in I\left(x_{i}\right)} W_{i j}\left(x_{i}^{(v)}-x_{j}^{(v)}\right)\right\|^{2}+\|\rho\|^{2} \\
&=\sum_{j, k \in I\left(x_{i}\right)} W_{i j} G_{j k}^{i} W_{i k}+\|\rho\|^{2} \\
& \text { s.t. } \quad\left\|W_{i .}\right\|=1, W_{i j} \geq 0,\|\rho\|_{1}=1, \rho_{v} \geq 0,
\end{aligned}
$$

where $\rho_{v}$ is used to balance the importance of different views, $W_{i}$. is the $i$-th row of $W, G_{j k}^{i}=\sum_{v}\left(\rho_{v} G_{j k}^{i(v)}\right), G_{j k}^{i(v)}$ is shown in Eq. 11 .

$$
G_{j k}^{i(v)}= \begin{cases}\left(x_{i}^{(v)}-x_{j}^{(v)}\right)^{T}\left(x_{i}^{(v)}-x_{k}^{(v)}\right), & j, k \in I\left(x_{i}\right) \\ 0, & \text { otherwise. }\end{cases}
$$

We iteratively solve $W_{i}$, and $\rho$. When a variable is fixed, the other one can be solved by using the entropic mirror descent algorithm (EMDA) Beck \& Teboulle (2003)). EMDA is provably a useful algorithm for solving large scale convex minimization problems over the unit simplex and is less dependent on the problem size while comparing with quadratic programming. The flow of EMDA is shown in Algorithm 1. Given $L_{f}$ and $f^{\prime}(x)$, the operations in EMDA are between scalars. Then, the computational complexity depends on the number of iterations and the length of vector. In our formulation, the length of vector is the number of views which is usually very small. Also, EMDA usually converges in limited iterations. While solving the vector with the unit simplex, the computational complexity mainly depends on calculating $L_{f}$ and $f^{\prime}(x)$.

The subgradients of $\epsilon_{i}$ with respect to $W_{i}$. and $\rho$ are in Eq. 12, where $\theta$ is $n_{v}$-dimensional and $\theta_{v}=W_{i .} G^{i(v)} W_{i .}^{T}$.

$$
\epsilon_{i}^{\prime}\left(W_{i .}\right)=W_{i .} G^{i} ; \quad \epsilon_{i}^{\prime}(\rho)=\theta+2 \rho
$$

EMDA needs a Lipschitz constant $L_{f}$. For a fixed norm (for example, 1norm), we get Eq. 13 and Eq.(14).

$$
L_{f}\left(W_{i .}\right)=\sup _{W_{i .}}\left\|\epsilon_{i}^{\prime}\left(W_{i .}\right)\right\|=\sup _{W_{i .}}\left\|W_{i .} G^{i}\right\|_{1}=\max _{s}\left(\sum_{t}\left|G_{s t}^{i}\right|\right)
$$




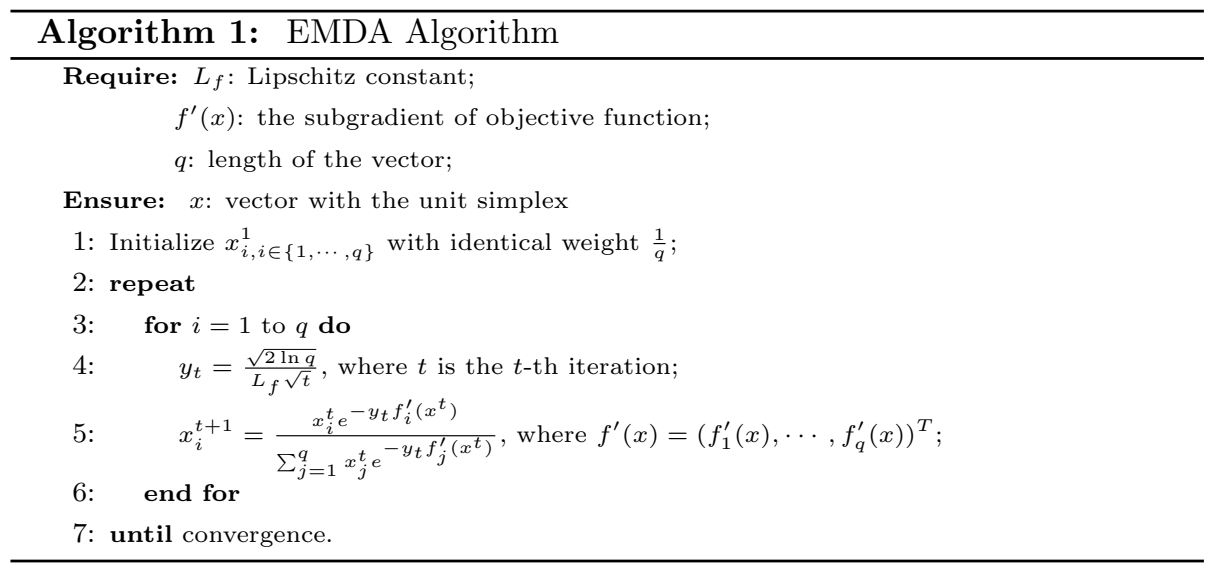

$$
L_{f}(\rho)=\sup _{\rho}\left\|\epsilon_{i}^{\prime}(\rho)\right\|=\sup _{\rho}\|\theta+2 \rho\|_{1}=\|\theta\|_{1}+2 .
$$

$W$ is asymmetric. We set the consensus adjacency matrix $A^{*}=\left(W+W^{T}\right) / 2$ which shows the locally geometrical structure in all views intuitively. Then, we can set the consensus manifold as $L^{*}=D^{*}-A^{*}, D_{i i}^{*}=\sum_{j} A_{i j}^{*}$.

\subsection{Constructing the consensus coefficient matrix}

\subsubsection{Linear combination}

In order to preserve the diverse geometrical structure of the low dimensional representations from different views, we can construct the consensus coefficient matrix $V^{*}$ convexly and linearly. That is, let $V^{*}$ be the linear combination of $V^{(v)}, V^{*}=\sum_{v=1}^{n_{v}} \alpha_{v} V^{(v)}, \alpha_{v} \geq 0, \sum_{v=1}^{n_{v}} \alpha_{v}=1$.

\subsubsection{Consensus matrix approximation}

Inspired by Liu et al. (2013), coefficient matrices representing multiple cluster structures should be regularized towards a common consensus matrix. The common consensus matrix is considered to reflect the latent cluster structure shared by different views. Thus we can use the regularization $\sum_{v} \alpha_{v} \| V^{*}-$ $V^{(v)} \|_{F}^{2}, \alpha_{v} \geq 0, \sum_{v=1}^{n_{v}} \alpha_{v}=1$ to obtain the consensus coefficient matrix $V^{*}$. 


\subsection{Four instances of the framework}

Combining different ways of finding consensus manifold and consensus coefficient, we get four instances of the MMNMF framework.

\subsection{1. $M M N M F-L-L$ algorithm}

Using linear combination to find the consensus manifold and consensus coefficient, the objective function is:

$$
\begin{array}{cc}
\Omega_{1}= & \sum_{v=1}^{n_{v}} D_{F}\left(X^{(v)} \| U^{(v)} V^{(v) T}\right)+\gamma\|\mu\|^{2}+\eta\|\alpha\|^{2} \\
+ & \lambda \operatorname{tr}\left(\left(\sum_{v=1}^{n_{v}} \alpha_{v} V^{(v)}\right)^{T}\left(\sum_{v=1}^{n_{v}} \mu_{v} L^{(v)}\right)\left(\sum_{v=1}^{n_{v}} \alpha_{v} V^{(v)}\right)\right) \\
\text { s.t. } \quad & U^{(v)} \geq 0, V^{(v)} \geq 0, V^{*} \geq 0 \\
& \mu=\left(\mu_{1}, \ldots, \mu_{n_{v}}\right)^{T}, \mu_{v} \geq 0, \sum_{v=1}^{n_{v}} \mu_{v}=1 \\
& \alpha=\left(\alpha_{1}, \ldots, \alpha_{n_{v}}\right)^{T}, \alpha_{v} \geq 0, \sum_{v=1}^{n_{v}} \alpha_{v}=1,
\end{array}
$$

where $\alpha_{v}$ and $\mu_{v}$ tune the relative weights among different views, $\lambda$ is used to control the contribution of the multi-manifold regularization, $\|\mu\|^{2}$ and $\|\alpha\|^{2}$ are used to avoid overfitting on one manifold and coefficient matrix, $\gamma$ and $\eta$ control the regularization term.

\subsection{2. $M M N M F-L-C$ algorithm}

Using the consensus coefficient in Liu et al. (2013) and the consensus manifold obtained through linear combination, the objective function is:

$$
\begin{array}{cc}
\Omega_{2}= & \sum_{v=1}^{n_{v}} D_{F}\left(X^{(v)} \| U^{(v)} V^{(v) T}\right)+\sum_{v=1}^{n_{v}} \alpha_{v}\left\|V^{*}-V^{(v)}\right\|_{F}^{2} \\
+ & \lambda \operatorname{tr}\left(V^{* T}\left(\sum_{v=1}^{n_{v}} \mu_{v} L^{(v)}\right) V^{*}\right)+\gamma\|\mu\|^{2}+\eta\|\alpha\|^{2} \\
\text { s.t. } & U^{(v)} \geq 0, V^{(v)} \geq 0, V^{*} \geq 0 \\
& \mu=\left(\mu_{1}, \ldots, \mu_{n_{v}}\right), \mu_{v} \geq 0, \sum_{v=1}^{n_{v}} \mu_{v}=1 \\
& \alpha=\left(\alpha_{1}, \ldots, \alpha_{n_{v}}\right)^{T}, \alpha_{v} \geq 0, \sum_{v=1}^{n_{v}} \alpha_{v}=1 .
\end{array}
$$




\subsection{3. $M M N M F-R-L$ algorithm}

Using multi-view LLE to find the consensus manifold and linear combination to find the consensus coefficient, the objective function is:

$$
\begin{aligned}
\Omega_{3}= & \sum_{v=1}^{n_{v}} D_{F}\left(X^{(v)} \| U^{(v)} V^{(v) T}\right) \\
& +\quad \lambda \operatorname{tr}\left(\left(\sum_{v=1}^{n_{v}} \alpha_{v} V^{(v)}\right)^{T}\right) L^{*}\left(\sum_{v=1}^{n_{v}} \alpha_{v} V^{(v)}\right)+\eta\|\alpha\|^{2} \\
& \text { s.t. } \quad U^{(v)} \geq 0, V^{(v)} \geq 0, V^{*} \geq 0 \\
& \alpha=\left(\alpha_{1}, \ldots, \alpha_{n_{v}}\right)^{T}, \alpha_{v} \geq 0, \sum_{v=1}^{n_{v}} \alpha_{v}=1 .
\end{aligned}
$$

\subsection{4. $M M N M F-R-C$ algorithm}

Using the consensus coefficient in Liu et al. (2013) and the consensus manifold obtained through multi-view LLE, the objective function is:

$$
\begin{aligned}
\Omega_{4}= & \sum_{v=1}^{n_{v}} D_{F}\left(X^{(v)} \| U^{(v)} V^{(v) T}\right)+\sum_{v=1}^{n_{v}} \alpha_{v}\left\|V^{*}-V^{(v)}\right\|_{F}^{2} \\
+ & \lambda \operatorname{tr}\left(V^{* T} L^{*} V^{*}\right)+\eta\|\alpha\|^{2} \\
\text { s.t. } & U^{(v)} \geq 0, V^{(v)} \geq 0, V^{*} \geq 0 \\
& \alpha=\left(\alpha_{1}, \ldots, \alpha_{n_{v}}\right)^{T}, \alpha_{v} \geq 0, \sum_{v=1}^{n_{v}} \alpha_{v}=1 .
\end{aligned}
$$

\subsection{Solution}

$\Omega$ is not jointly convex, but convex for each variable individually. So we present an iterative algorithm which optimizes one variable while fixing others to minimize $\Omega$. The solutions of the four instances are similar, we give the details of MMNMF-L-L and report the results of others only.

\subsubsection{Solution of $M M N M F-L-L$}

It is easy to see that the solution of $U^{(v)}$ is the same with that of NMF. As suggested in Lee \& Seung (2001), we can achieve $U^{(v)}$ by iteratively updating 
Eq. 19. .

$$
U^{(v)} \longleftarrow U^{(v)} \odot \frac{X^{(v)} V^{(v)}}{U^{(v)} V^{(v) T} V^{(v)}},
$$

where : denotes matrix element-wise division, $\odot$ denotes matrix element-wise multiplication.

Let $\Psi^{(v)}$ be the Lagrange multiplier for $V^{(v)}$, then the Lagrange function of $\Omega_{1}$ can be expressed by

$$
\Gamma=\Omega_{1}+\sum_{v=1}^{n_{v}} \operatorname{tr}\left(\Psi^{(v)} V^{(v) T}\right)
$$

Deducing the first-order derivative for $V^{(v)}$, we get

$$
\begin{aligned}
\frac{\partial \Gamma}{\partial V^{(v)}} & =-2 X^{(v) T} U^{(v)}+2 V^{(v)} U^{(v) T} U^{(v)}+\Psi^{(v)} \\
& +2 \lambda \alpha_{v}\left(\sum_{k=1}^{n_{v}} \mu_{k} L^{(k)}\right)\left(\sum_{k=1}^{n_{v}} \alpha_{k} V^{(k)}\right) .
\end{aligned}
$$

Using the Karush-Kuhn-Tucker conditions (Boyd \& Vandenberghe (2004)), we can get the update rule:

$$
V^{(v)} \longleftarrow V^{(v)} \odot \frac{X^{(v) T} U^{(v)}+\lambda \alpha_{v} A^{*} V^{*}}{V^{(v)} U^{(v) T} U^{(v)}+\lambda \alpha_{v} D^{*} V^{*}},
$$

where $V^{*}=\sum_{k=1}^{n_{v}} \alpha_{k} V^{(k)}, A^{*}=\sum_{k=1}^{n_{v}} \mu_{k} A^{(k)}$ and $D^{*}=\sum_{k=1}^{n_{v}} \mu_{k} D^{(k)}$.

To optimize manifold parameter $\mu$ and coefficient matrix parameter $\alpha$, we can use the entropic mirror descent algorithm (EMDA) (Beck \& Teboulle (2003)). 
While updating $\alpha$, we fix the others and get the following objective function:

$$
\begin{aligned}
f(\alpha)= & \lambda \operatorname{tr}\left(\left(\sum_{v=1}^{n_{v}} \alpha_{v} V^{(v) T}\right) L^{*}\left(\sum_{v=1}^{n_{v}} \alpha_{v} V^{(v)}\right)\right)+\eta\|\alpha\|^{2} \\
= & \lambda \sum_{i, j=1}^{n_{v}} \alpha_{i} \alpha_{j} \operatorname{tr}\left(V^{(i) T} L^{*} V^{(j)}\right)+\eta\|\alpha\|^{2} \\
= & \alpha^{T}(\lambda B+\eta I) \alpha \\
\text { s.t. } \quad & L^{*}=\sum_{v=1}^{n_{v}} \mu_{v} L^{(v)} \\
& B_{i j}=\operatorname{tr}\left(V^{(i) T} L^{*} V^{(j)}\right) \\
& \alpha=\left(\alpha_{1}, \ldots, \alpha_{n_{v}}\right)^{T}, \alpha_{v} \geq 0, \sum_{v=1}^{n_{v}} \alpha_{v}=1 .
\end{aligned}
$$

For a fixed norm (for example 1-norm), the subgradient and Lipschitz constant of $\alpha$ are shown in Eq.24 and Eq.25.

$$
\begin{gathered}
f^{\prime}(\alpha)=(2 \lambda B+2 \eta I) \alpha . \\
L_{f}(\alpha)=\sup _{\alpha}\left\|f^{\prime}(\alpha)\right\|_{1}=\max _{j}\left|\sum_{i}(2 \lambda B+2 \eta I)_{i j}\right| .
\end{gathered}
$$

While updating $\mu$, we fix the others and get the following objective function:

$$
\begin{aligned}
f(\mu)= & \lambda \operatorname{tr}\left(V^{* T}\left(\sum_{v=1}^{n_{v}} \mu_{v} L^{(v)}\right) V^{*}\right)+\gamma\|\mu\|^{2} \\
= & \lambda \sum_{v=1}^{n_{v}} \mu_{v} b_{v}+\gamma\|\mu\|^{2} \\
\text { s.t. } \quad & V^{*}=\sum_{v=1}^{n_{v}} \alpha_{v} V^{(v)} \\
& b_{v}=\operatorname{tr}\left(V^{* T} L^{(v)} V^{*}\right) \\
& \mu=\left(\mu_{1}, \ldots, \mu_{n_{v}}\right)^{T}, \mu_{v} \geq 0, \sum_{v=1}^{n_{v}} \mu_{v}=1 .
\end{aligned}
$$

For a fixed norm (for example 1-norm), the subgradient and Lipschitz constant of $\mu$ are shown in Eq. 27].

$$
f^{\prime}(\mu)=\lambda b+2 \gamma \mu ; \quad L_{f}(\mu)=\sup _{\mu}\left\|f^{\prime}(\mu)\right\|_{1}=\lambda\|b\|_{1}+2 \gamma .
$$




\subsubsection{Solution of $M M N M F-L-C$}

The updating of $U^{(v)}$ is the same with that of MMNMF-L-L.

$$
\begin{gathered}
V^{(v)} \longleftarrow V^{(v)} \odot \frac{X^{(v) T} U^{(v)}+\alpha_{v} V^{*}}{V^{(v)} U^{(v) T} U^{(v)}+\alpha_{v} V^{(v)}}, \\
V^{*} \longleftarrow V^{*} \odot \frac{\sum_{v=1}^{n_{v}} \alpha_{v} V^{(v)}+\lambda A^{*} V^{*}}{\sum_{v=1}^{n_{v}} \alpha_{v} V^{*}+\lambda D^{*} V^{*}},
\end{gathered}
$$

where $A^{*}=\sum_{k=1}^{n_{v}} \mu_{k} A^{(k)}, D^{*}=\sum_{k=1}^{n_{v}} \mu_{k} D^{(k)}$.

While updating $\alpha, f^{\prime}(\alpha)=b+2 \eta \alpha, L_{f}(\alpha)=\|b\|_{1}+2 \eta$, where $b_{v}=\| V^{*}-$ $V^{(v)} \|_{F}^{2}$.

While updating $\mu, f^{\prime}(\mu)=2 \lambda b+2 \gamma \mu, L_{f}(\mu)=\lambda\|b\|_{1}+2 \gamma$, where $b_{v}=$ $\operatorname{tr}\left(V^{* T} L^{(v)} V^{*}\right)$.

\subsubsection{Solution of $M M N M F-R-L$}

The updating of $U^{(v)}$ is the same with that of MMNMF-L-L.

$$
V^{(v)} \longleftarrow V^{(v)} \odot \frac{X^{(v) T} U^{(v)}+\lambda \alpha_{v} A^{*} V^{*}}{V^{(v)} U^{(v) T} U^{(v)}+\lambda \alpha_{v} D^{*} V^{*}},
$$

where $V^{*}=\sum_{k=1}^{n_{v}} \alpha_{k} V^{(k)}, A^{*}=\left(W+W^{T}\right) / 2, D_{i i}^{*}=\sum_{j} A_{i j}^{*}, W$ is calculated through multi-view LLE.

While updating $\left.\alpha, f^{\prime} \alpha\right)=(2 \lambda B+2 \eta I) \alpha, L_{f}(\alpha)=\max _{j}\left|\sum_{i}(2 \lambda B+2 \eta I)_{i j}\right|$, where $B_{i j}=\operatorname{tr}\left(V^{(i) T}\left(D^{*}-A^{*}\right) V^{(j)}\right)$.

\subsubsection{Solution of $M M N M F-R-C$}

The updating of $U^{(v)}$ is the same with that of MMNMF-L-L.

$$
\begin{gathered}
V^{(v)} \longleftarrow V^{(v)} \odot \frac{X^{(v) T} U^{(v)}+\alpha_{v} V^{*}}{V^{(v)} U^{(v) T} U^{(v)}+\alpha_{v} V^{(v)}}, \\
V^{*} \longleftarrow V^{*} \odot \frac{\sum_{v=1}^{n_{v}} \alpha_{v} V^{(v)}+\lambda A^{*} V^{*}}{\sum_{v=1}^{n_{v}} \alpha_{v} V^{*}+\lambda D^{*} V^{*}},
\end{gathered}
$$

$255 \quad$ where $A^{*}=\left(W+W^{T}\right) / 2, D_{i i}^{*}=\sum_{j} A_{i j}^{*}, W$ is calculated through multi-view LLE.

While updating $\alpha, f^{\prime}(\alpha)=b+2 \eta \alpha, L_{f}(\alpha)=\|b\|_{1}+2 \eta$, where $b_{v}=\| V^{*}-$ $V^{(v)} \|_{F}^{2}$. 


\subsection{Convergence}

260 be converged. We prove the convergence of the rules of updating $V^{(v)}$ and $V^{*}$. Since the proofs of the four instances are nearly the same, we give the details of MMNMF-L-C only. At first, we introduce the definition and lemma of auxiliary function (Lee \& Seung (2001)). We refer readers to a prior reading of the paper

Definition 1. $F\left(V, V^{t}\right)$ is an auxiliary function for $\Omega(V)$ if the conditions

$$
F\left(V, V^{t}\right) \geq \Omega(V), F\left(V^{t}, V^{t}\right)=\Omega\left(V^{t}\right)
$$

are satisfied. $V^{t}$ is the value of $V$ in $t$-th iteration.

Lemma 1. If $F$ is an auxiliary function, then $\Omega(V)$ is non-increasing under the update rule

$$
V^{t+1}=\arg \min _{V} F\left(V, V^{t}\right)
$$

The key point is to find an appropriate auxiliary function for $\Omega_{2}$. Since the update rule is element-wise, we just need to prove that $\Omega_{2}$ is non-increasing under the update rule of $V_{i j}^{(v)}$ and $V_{i j}^{*}$. That is, we need to prove the following two propositions.

- Fixing $U^{(v)}$ and $V^{*}, \Omega_{2}$ is non-increasing under the update rule 28);

- Fixing $U^{(v)}$ and $V^{(v)}, \Omega_{2}$ is non-increasing under the update rule 29 .

\subsubsection{Convergence of updating $V^{(v)}$}

Let $\Omega_{2}\left(V^{(v)}\right)=\left\|X^{(v)}-U^{(v)} V^{(v) T}\right\|_{F}^{2}+\alpha_{v}\left\|V^{*}-V^{(v)}\right\|_{F}^{2}$ be the parts of $\Omega_{2}$ 275

containing $V^{(v)}$. Then, $F\left(V_{i j}^{(v)}, V_{i j}^{(v) t}\right)$ in Eq. 35 is an auxiliary function for $\Omega_{2}\left(V^{(v)}\right)$.

$$
F\left(V_{i j}^{(v)}, V_{i j}^{(v) t}\right)=\Omega_{2}\left(V_{i j}^{(v) t}\right)+\Omega_{2}^{\prime}\left(V_{i j}^{(v) t}\right)\left(V_{i j}^{(v)}-V_{i j}^{(v) t}\right)+\Phi\left(V_{i j}^{(v) t}\right)\left(V_{i j}^{(v)}-V_{i j}^{(v) t}\right)^{2}
$$


where

$$
\begin{gathered}
\Omega_{2}^{\prime}\left(V_{i j}^{(v) t}\right)=\left(-2 X^{(v) T} U^{(v)}+2 V^{(v) t} U^{(v) T} U^{(v)}+2 \alpha_{v} V^{(v) t}-2 \alpha_{v} V^{*}\right)_{i j}, \\
\Phi\left(V_{i j}^{(v) t}\right)=\frac{\left(V^{(v) t} U^{(v) T} U^{(v)}\right)_{i j}+\alpha_{v} V_{i j}^{(v) t}}{V_{i j}^{(v) t}} .
\end{gathered}
$$

When $V_{i j}^{(v)}=V_{i j}^{(v) t}, F\left(V_{i j}^{(v)}, V_{i j}^{(v) t}\right)=\Omega_{2}\left(V_{i j}^{(v)}\right)$ is obvious. We only need to show $F\left(V_{i j}^{(v)}, V_{i j}^{(v) t}\right) \geq \Omega_{2}\left(V_{i j}^{(v)}\right)$ otherwise. To do this, we compare the Taylor series expansion of $\Omega_{2}\left(V_{i j}^{(v)}\right)$ (Eq. (36)) with $F\left(V_{i j}^{(v)}, V_{i j}^{(v) t}\right.$ ).

$$
\Omega_{2}\left(V_{i j}^{(v)}\right)=\Omega_{2}\left(V_{i j}^{(v) t}\right)+\Omega_{2}^{\prime}\left(V_{i j}^{(v) t}\right)\left(V_{i j}^{(v)}-V_{i j}^{(v) t}\right)+\frac{\Omega_{2}^{\prime \prime}\left(V_{i j}^{(v) t}\right)}{2}\left(V_{i j}^{(v)}-V_{i j}^{(v) t}\right)^{2},
$$

where

$$
\Omega_{2}^{\prime \prime}\left(V_{i j}^{(v) t}\right)=2\left(U^{(v) T} U^{(v)}\right)_{j j}+2 \alpha_{v} .
$$

We find that proving $F\left(V_{i j}^{(v)}, V_{i j}^{(v) t}\right) \geq \Omega_{2}\left(V_{i j}^{(v)}\right)$ is equivalent to proving Eq. (37).

$$
\left.\Phi\left(V_{i j}^{(v) t}\right) \geq \frac{\Omega_{2}^{\prime \prime}\left(V_{i j}^{(v) t}\right)}{2}\right) .
$$

As

$$
\left(V^{(v) t} U^{(v) T} U^{(v)}\right)_{i j}=\sum_{l=1}^{r} V_{i l}^{(v) t}\left(U^{(v) T} U^{(v)}\right)_{l j} \geq V_{i j}^{(v) t}\left(U^{(v) T} U^{(v)}\right)_{j j},
$$

thus Eq. 377 holds. According to Eq. (34),

$$
\begin{aligned}
V_{i j}^{(v)} & =-\frac{-2 \Phi\left(V_{i j}^{(v) t}\right) V_{i j}^{(v) t}+\Omega_{2}^{\prime}\left(V_{i j}^{(v) t}\right)}{2 \Phi\left(V_{i j}^{(v) t}\right)} \\
& =V_{i j}^{(v) t} \frac{\left(X^{(v) T} U^{(v)}+\alpha_{v} V^{*}\right)_{i j}}{\left(V^{(v) t} U^{(v) T} U^{(v)}+\alpha_{v} V^{(v) t}\right)_{i j}} .
\end{aligned}
$$

\subsubsection{Convergence of updating $V^{*}$}

Let $\Omega_{2}\left(V^{*}\right)=\sum_{v=1}^{n_{v}} \alpha_{v}\left\|V^{*}-V^{(v)}\right\|_{F}^{2}+\lambda \operatorname{tr}\left(V^{* T}\left(\sum_{v=1}^{n_{v}} \mu_{v} L^{(v)}\right) V^{*}\right)$ be the parts of $\Omega_{2}$ containing $V^{*}$. Then, $F\left(V_{i j}^{*}, V_{i j}^{* t}\right)$ in Eq. 38 is an auxiliary function for $\Omega_{2}\left(V^{*}\right)$.

$$
F\left(V_{i j}^{*}, V_{i j}^{* t}\right)=\Omega_{2}\left(V_{i j}^{* t}\right)+\Omega_{2}^{\prime}\left(V_{i j}^{* t}\right)\left(V_{i j}^{*}-V_{i j}^{* t}\right)+\Phi\left(V_{i j}^{* t}\right)\left(V_{i j}^{*}-V_{i j}^{* t}\right)^{2},
$$


where

$$
\begin{gathered}
\Omega_{2}^{\prime}\left(V_{i j}^{* t}\right)=\sum_{v=1}^{n_{v}} 2 \alpha_{v}\left(V^{* t}-V^{(v)}\right)_{i j}+2 \lambda\left(\left(\sum_{v=1}^{n_{v}} \mu_{v} L^{(v)}\right) V^{* t}\right)_{i j}, \\
\Phi\left(V_{i j}^{* t}\right)=\sum_{v=1}^{n_{v}} \alpha_{v}+\lambda\left(\sum_{v=1}^{n_{v}} \mu_{v} D_{i j}^{(v)}\right) .
\end{gathered}
$$

When $V_{i j}^{*}=V_{i j}^{* t}, F\left(V_{i j}^{*}, V_{i j}^{* t}\right)=\Omega_{2}\left(V_{i j}^{*}\right)$ is obvious. We only need to show $F\left(V_{i j}^{*}, V_{i j}^{* t}\right) \geq \Omega_{2}\left(V_{i j}^{*}\right)$ otherwise. To do this, we compare the Taylor series expansion of $\Omega_{2}\left(V_{i j}^{*}\right)$ (Eq. $\left.39 p\right)$ with $F\left(V_{i j}^{*}, V_{i j}^{* t}\right)$.

$$
\Omega_{2}\left(V_{i j}^{*}\right)=\Omega_{2}\left(V_{i j}^{* t}\right)+\Omega_{2}^{\prime}\left(V_{i j}^{* t}\right)\left(V_{i j}^{*}-V_{i j}^{* t}\right)+\frac{\Omega_{2}^{\prime \prime}\left(V_{i j}^{* t}\right)}{2}\left(V_{i j}^{*}-V_{i j}^{* t}\right)^{2},
$$

where

$$
\Omega_{2}^{\prime \prime}\left(V_{i j}^{* t}\right)=2 \sum_{v=1}^{n_{v}} \alpha_{v}+2 \lambda\left(\sum_{v=1}^{n_{v}} \mu_{v}\left(D^{(v)}-A^{(v)}\right)_{i j}\right) .
$$

We find that proving $F\left(V_{i j}^{*}, V_{i j}^{* t}\right) \geq \Omega_{2}\left(V_{i j}^{*}\right)$ is equivalent to proving Eq.40.

$$
\left.\Phi\left(V_{i j}^{* t}\right) \geq \frac{\Omega_{2}^{\prime \prime}\left(V_{i j}^{* t}\right)}{2}\right) .
$$

As

$$
\sum_{v=1}^{n_{v}} \mu_{v} D_{i j}^{(v)} \geq \sum_{v=1}^{n_{v}} \mu_{v}\left(D^{(v)}-A^{(v)}\right)_{i j},
$$

thus Eq.40 holds. According to Eq. 34,

$$
\begin{aligned}
V_{i j}^{*} & =-\frac{-2 \Phi\left(V_{i j}^{* t}\right) V_{i j}^{* t}+\Omega_{2}^{\prime}\left(V_{i j}^{* t}\right)}{2 \Phi\left(V_{i j}^{* t}\right)} \\
& =V_{i j}^{* t} \frac{\left(\sum_{v=1}^{n_{v}} \alpha_{v} V^{(v)}+\lambda \sum_{v=1}^{n_{v}}\left(\mu_{v} A^{(v)}\right) V^{* t}\right)_{i j}}{\left(\sum_{v=1}^{n_{v}} \alpha_{v} V^{* t}+\lambda \sum_{v=1}^{n_{v}}\left(\mu_{v} D^{(v)}\right) V^{* t}\right)_{i j}} .
\end{aligned}
$$

\subsection{The algorithm}

The flow of MMNMF is shown in Algorithm2(MMNMF-L-L and MMNMFR-L) and Algorithm 3 (MMNMF-L-C and MMNMF-R-C). Since MMNMFL-C and MMNMF-R-C find a consensus $V^{*}$ in the process of iteration, we implement them by using the method in MultiNMF (Liu et al. (2013)), i.e., we conduct several internal iterations of $V^{(v)}$ and $U^{(v)} . \lambda$ controls the contribution of manifold regularization. An appropriate $\lambda$ varies with dataset and algorithm. 

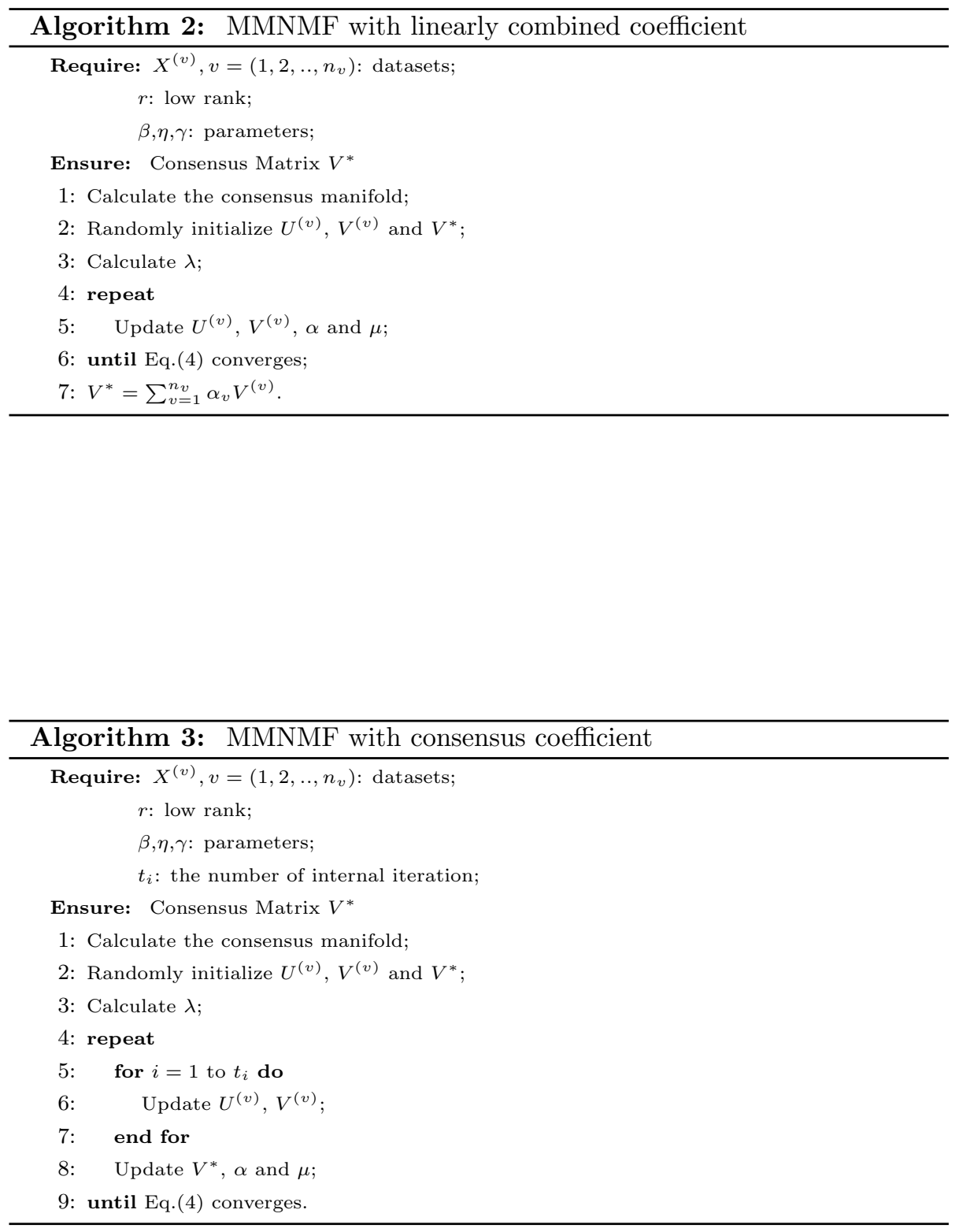
Table 1: Computational complexities of the proposed algorithms.

\begin{tabular}{|c|c|c|c|c|}
\hline & MMNMF-L-L & MMNMF-L-C & MMNMF-R-L & MMNMF-R-C \\
\hline$L^{*}$ & $O\left(n^{2} m n_{v}\right)$ & $O\left(n^{2} m n_{v}\right)$ & $O\left(n n^{\prime} m n_{v}\right)$ & $O\left(n n^{\prime} m n_{v}\right)$ \\
\hline$U^{(v)}$ & $O\left(m^{(v)} n r\right)$ & $O\left(m^{(v)} n r\right)$ & $O\left(m^{(v)} n r\right)$ & $O\left(m^{(v)} n r\right)$ \\
\hline$V^{(v)}$ & $O\left(n^{2} r+m^{(v)} n r\right)$ & $O\left(m^{(v)} n r\right)$ & $O\left(n^{2} r+m^{(v)} n r\right)$ & $O\left(m^{(v)} n r\right)$ \\
\hline$V^{*}$ & $O\left(n^{2} n_{v}\right)$ & $O\left(n^{2} r\right)$ & $O\left(n^{2} n_{v}\right)$ & $O\left(n^{2} r\right)$ \\
\hline$\mu$ & $O\left(n^{2} n_{v} r\right)$ & $O\left(n^{2} n_{v} r\right)$ & - & - \\
\hline$\alpha$ & $O\left(n^{2} n_{v}^{2} r\right)$ & $O\left(n n_{v} r\right)$ & $O\left(n^{2} n_{v}^{2} r\right)$ & $O\left(n n_{v} r\right)$ \\
\hline
\end{tabular}

We should adjust $\lambda$ according to dataset and algorithm. Firstly, MMNMF iterates $U^{(v)}, V^{(v)}$ and $V^{*}$ once with $\lambda=\beta$. Then, we set $\lambda$ as Eq.41) to balance NMF and manifold regularization. We do not use the proportion initially due to the fact that the random initialization cannot reflect the real relation between NMF and manifold regularization.

$$
\lambda=\beta * \frac{\sum_{v=1}^{n_{v}} D\left(X^{(v)} \| U^{(v)} V^{(v) T}\right)}{\operatorname{tr}\left(V^{* T} L^{*} V^{*}\right)} .
$$

\subsection{Complexity analysis}

In MMNMF, the main part is calculating $L^{*}, U^{(v)}, V^{(v)}, V^{*}, \mu$ and $\alpha$. Let $n$ be the number of data points, $n_{v}$ be the number of views, $m^{(v)}$ be the dimension of the $v$-th view, $m=\max _{v} m^{(v)}, r$ be the low rank, $n_{I}^{i}=\left|I\left(x_{i}\right)\right|$ be the total number of $x_{i}$ 's neighbors, $n_{I}=\max _{i} n_{I}^{i}, n^{\prime}=\max \left\{n, n_{I}^{3}\right\}$. The detailed computational complexities of the proposed algorithms are shown in Table 1. For MMNMF-L-L and MMNMF-L-C, the complexity of calculating $L^{*}$ is $O\left(n^{2} m n_{v}\right)$ which includes the complexity of linear combination and the complexity of calculating each view's Laplacian matrix. For MMNMF-R-L and MMNMF-R-C, calculating $L^{*}$ mainly includes calculating the neighbors, the Lipschitz constant and the subgradient. The complexity of calculating $\mu$ and $\alpha$ mainly includes the complexity of calculating the Lipschitz constant and the subgradient. The complexity of calculating $U^{(v)}, V^{(v)}, V^{*}$ is the complexity of the corresponding updating rules. 


\section{Experiments}

\subsection{Datasets}

We experiment on six benchmark datasets. The details are list in Table 2.

Webkb 1 is composed of web pages collected from computer science depart-

\subsection{Baselines}

We compare the proposed four instances with the following algorithms: single view NMF (SNMF) (Lee \& Seung (1999)), single view graph regularized NMF (SGNMF) (Cai et al. (2011)), feature concatenation (ConcatNMF), collec-

\footnotetext{
${ }^{1}$ http://www.cs.cmu.edu/afs/cs.cmu.edu/project/theo-20/www/data/

${ }^{2}$ http://mlg.ucd.ie/datasets/3sources.html

${ }^{3}$ https://archive.ics.uci.edu/ml/datasets/Multiple+Features

${ }^{4}$ http://lig-membres.imag.fr/grimal/data.html

${ }^{5}$ http://mlg.ucd.ie/aggregation/index.html

${ }^{6}$ http://elki.dbs.ifi.lmu.de/wiki/DataSets/MultiView
} 
Table 2: Statistics of the datasets

\begin{tabular}{|l|c|c|c|}
\hline Dataset & \# of Instances & \# of Views & \# of Classes \\
\hline Webkb & 226 & 2 & 4 \\
3Sources & 169 & 3 & 6 \\
Handwritten & 2000 & 2 & 10 \\
Cora & 2708 & 2 & 7 \\
Politics-uk & 419 & 9 & 5 \\
ALOI & 11025 & 2 & 100 \\
\hline
\end{tabular}

NMF (MultiNMF) (Liu et al. (2013)) and multi-view graph regularized NMF (MultiGNMF). ConcatNMF constructs new data matrix by concatenating the features of all the views. MultiGNMF combines GNMF with multi-view clustering and its objective function is Eq.42,

$$
\begin{aligned}
\Theta & =\sum_{v=1}^{n_{v}} D_{F}\left(X^{(v)} \| U^{(v)} V^{(v) T}\right) \\
& +\lambda \sum_{v=1}^{n_{v}} \operatorname{tr}\left(V^{(v) T} L^{(v)} V^{(v)}\right) \\
& +\sum_{v=1}^{n_{v}}\left\|V^{*}-V^{(v)}\right\|_{F}^{2}
\end{aligned}
$$

We adopt the Frobenius norm to measure the difference between the original samples and their approximations for all algorithms.

\subsection{Evaluation metric}

We employ accuracy (ACC) and normalized mutual information (NMI) to evaluate clustering performance (Xu et al. (2003)).

The accuracy (ACC) measure calculates the largest rate of correct assignation by corresponding each data point to the right cluster. Denote $c_{i}$ as the label of an algorithm and $l_{i}$ as the true label of $x_{i}$. ACC is defined as:

$$
A C C=\frac{\sum_{i=1}^{n} \delta\left(l_{i}, \operatorname{map}\left(c_{i}\right)\right)}{n},
$$

where $\delta(x, y)$ is the indicator function, and $n$ is the total number of data points, $\operatorname{map}\left(c_{i}\right)$ is the mapping function that permutes the clustering labels to match the ground truth labels. 


\subsubsection{Normalized mutual information}

The normalized mutual information(NMI) indicates the shared statistics between the label of an algorithm and the true label. Given the true labels $\Delta=\left\{C_{1}, C_{2}, \ldots, C_{c}\right\}$ and the clustering results $\Delta^{\prime}=\left\{C_{1}^{\prime}, C_{2}^{\prime}, \ldots, C_{k}^{\prime}\right\}$ of $X(|X|=n)$, let $n_{i}$ and $n_{i}^{\prime}$ be the number of data points in cluster $C_{i}$ and $C_{i}^{\prime}$ separately. Let $n_{s t}$ denote the number of data points that are in cluster $C_{s}$ as well as in cluster $C_{t}^{\prime}$, then the normalized mutual information of $\Delta$ and $\Delta^{\prime}$ is

$$
\mathrm{NMI}=\frac{\sum_{s=1}^{c} \sum_{t=1}^{k} \log \left(\frac{n n_{s t}}{n_{s} n_{t}^{\prime}}\right)}{\sqrt{\left(\sum_{s=1}^{c} n_{s} \log \frac{n_{s}}{n}\right)\left(\sum_{t=1}^{k} n_{t}^{\prime} \log \frac{n_{t}^{\prime}}{n}\right)}} .
$$

345

\subsection{Experimental settings}

There are three parameters in Algorithm 2 and Algorithm 3. $\lambda$ controls the contribution of the manifold regularization. $\gamma$ and $\eta$ control the regularization avoiding overfitting. For MultiGNMF and SGNMF, we use the same method investigate the influence of $\beta, \gamma, \eta$ and tune one parameter by fixing the others.

Since $\gamma$ and $\eta$ are often set as 1 , we firstly set $\gamma$ and $\eta$ as 1 , and make $\beta$ range from $10^{-3}$ to $10^{3}$. Figure 1 and Figure 2 show the variation of ACC and NMI on the datasets with different $\beta$. In most cases, MMNMF-L-L and

SGNMF perform well when $\beta \in[6,1000]$. The performance of MMNMF-L-C is stable when $\beta \in[0.4,2]$. MMNMF-R-L has relatively better performance when $\beta \in[2,6]$. MMNMF-R-C performs well when $\beta \in[0.8,2]$. The performance of MultiGNMF is relatively better when $\beta \in[6,10]$. In the following experiments, we set $\beta=8$ for MultiGNMF and SGMF, $\beta=10$ for MMNMF-L-L, $\beta=1$ for MMNMF-R-C, $\beta=2$ for MMNMF-R-L and $\beta=0.8$ for MMNMF-L-C.

Then we experiment on $\eta$ with $\gamma=1$ and the above suggestions of $\beta$. The ACC and NMI on different datasets with $\eta$ ranging from $10^{-3}$ to $10^{3}$ are shown in Figure 3 and Figure 4 The algorithms perform stably when $\eta$ ranges from $10^{-3}$ to $10^{2}$ on all datasets. In the following experiments, we set $\eta$ as 1 for all 365 the algorithms. 


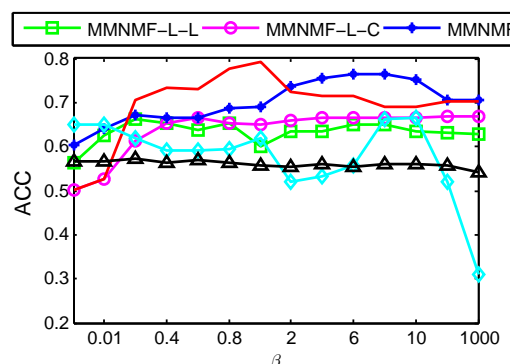

(a) Webkb

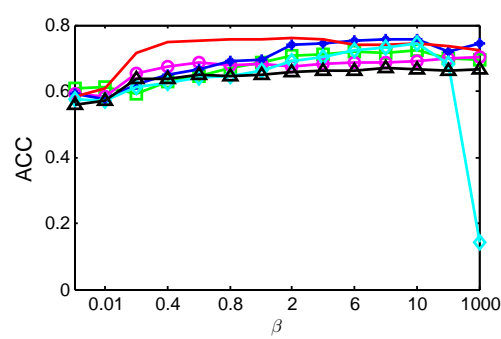

(c) Handwriten

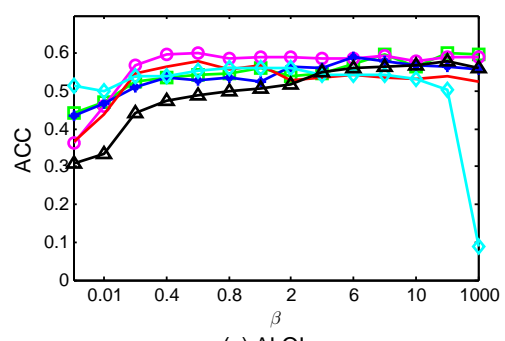

(e) ALOI

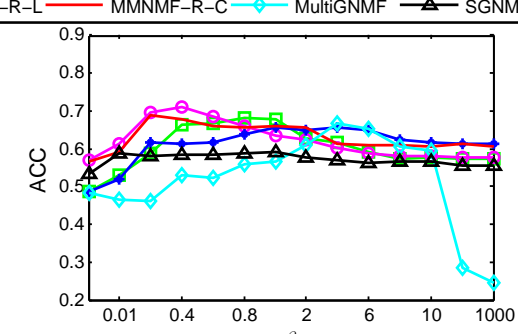

(b) 3 Sources
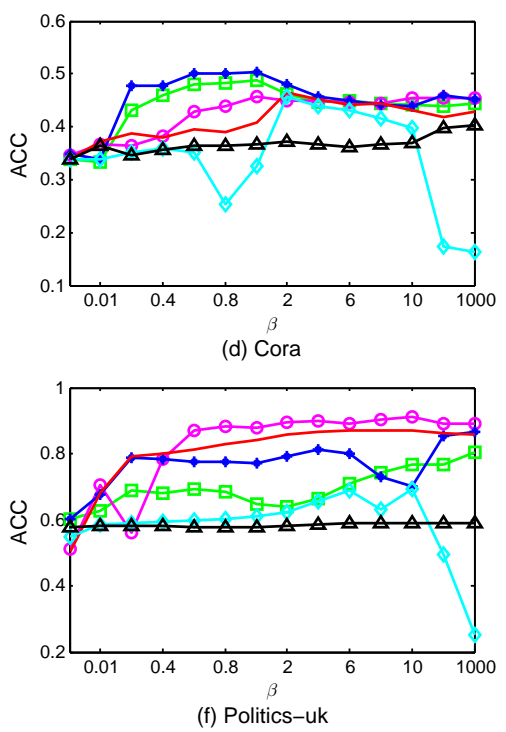

Figure 1: ACC of manifold based algorithms on all datasets with various $\beta$ 


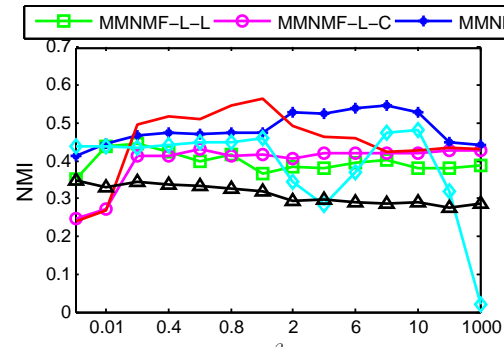

(a) Webkb

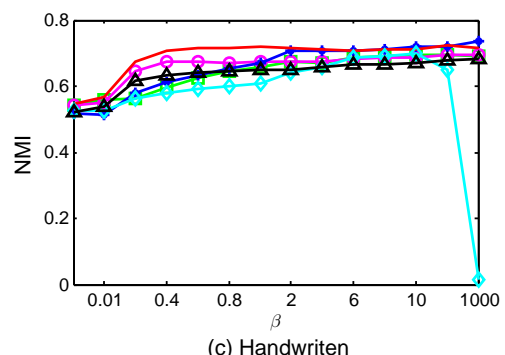

(c) Handwriten

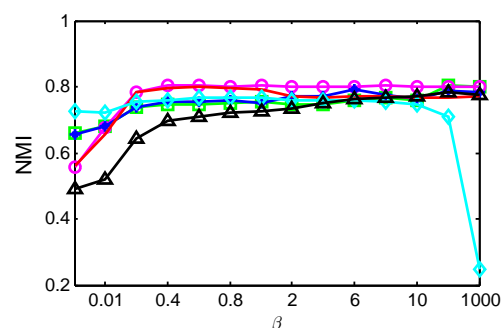

(e) ÁLOI

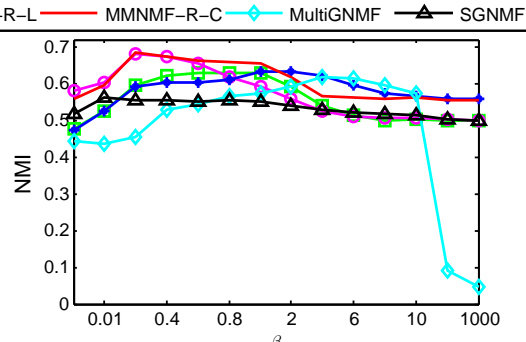

(b) 3Sources

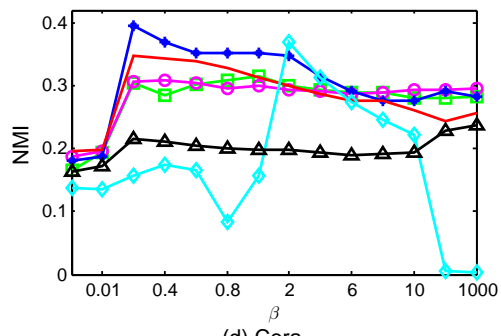

(d) Cora

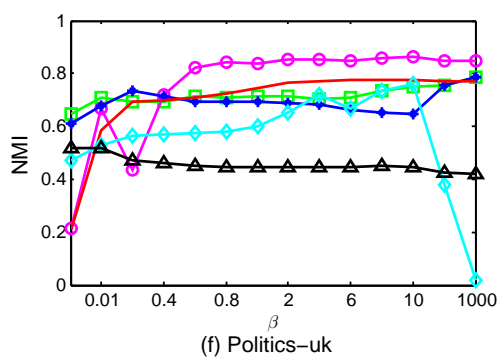

Figure 2: NMI of manifold based algorithms on all datasets with various $\beta$

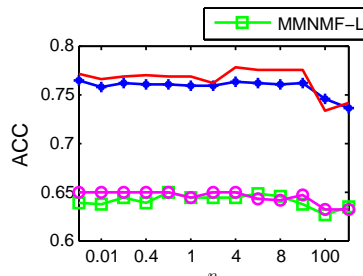

(a) Webkb

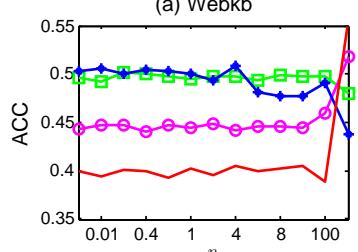

(d) Cora

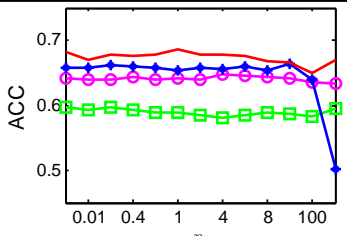

(b) 3 Sources

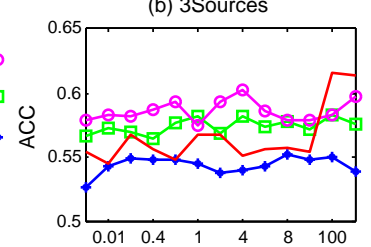

(e) ALOI

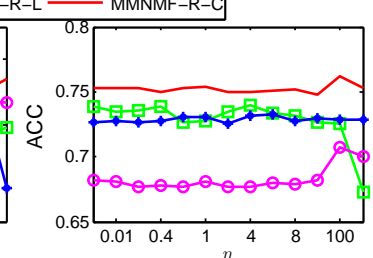

(c) Handwriten

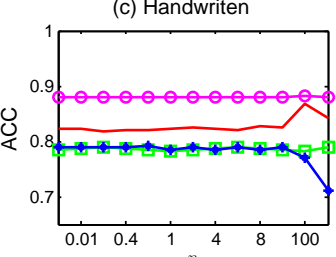

(f) Politics-uk

Figure 3: ACC of manifold based algorithms on all datasets with various $\eta$. 

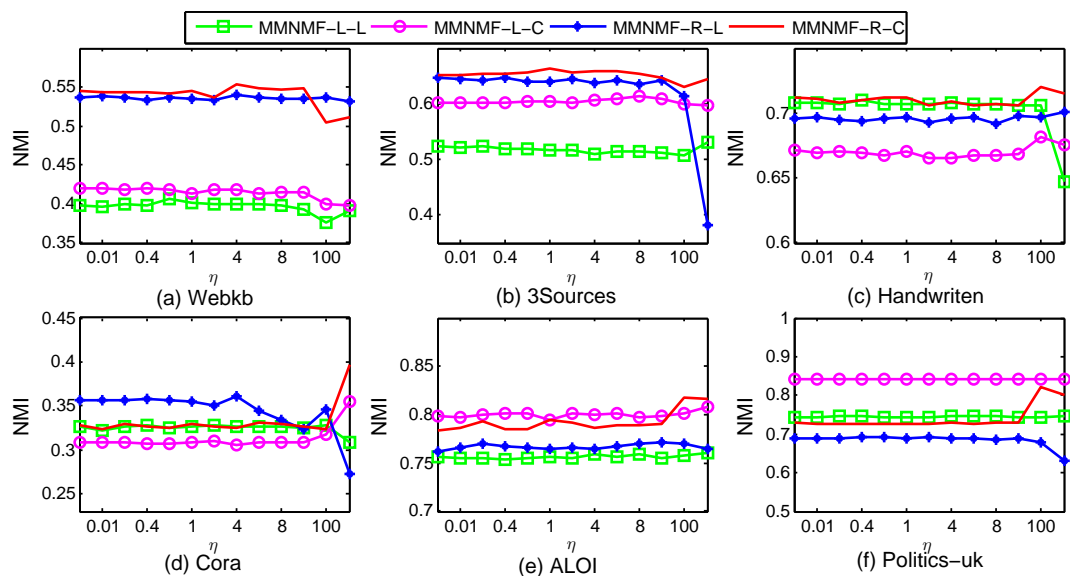

Figure 4: NMI of manifold based algorithms on all datasets with various $\eta$.
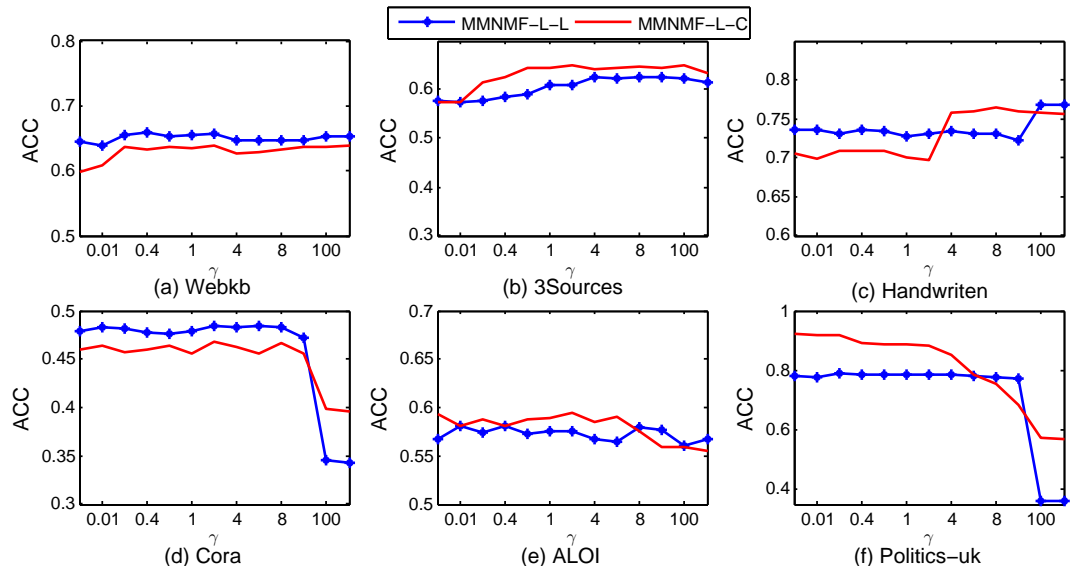

Figure 5: ACC of manifold based algorithms on all datasets with various $\gamma$. 


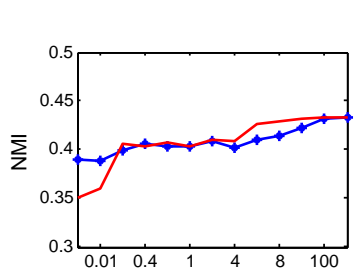

(a) Webkb

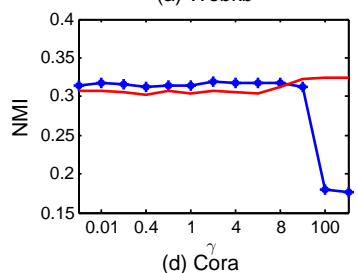

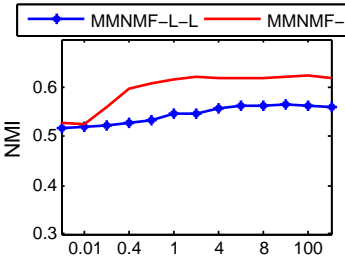

(b) 3 Sources

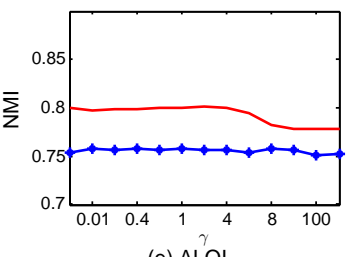

(e) ALOI

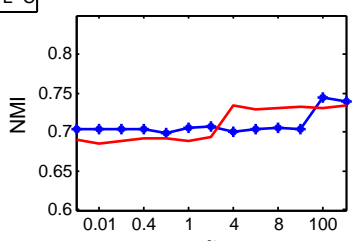

(c) Handwriten

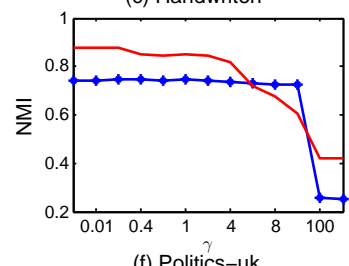

(f) Politics-uk

Figure 6: NMI of manifold based algorithms on all datasets with various $\gamma$.

Finally, we experiment on $\gamma$ with the above suggestions of $\beta$ and $\eta$. The ACC and NMI on different datasets with $\gamma$ ranging from $10^{-3}$ to $10^{3}$ are shown in Figure 5 and Figure 6. MMNMF-L-L performs stably when $\gamma$ ranges from $10^{-3}$ to 8 on all datasets. MMNMF-L-C performs stably when $\gamma$ ranges from 0.4 to 4 on Webkb, 3Sources, Cora, ALOI. The performance of MMNMF-L$\mathrm{C}$ decreases with the increasing of $\gamma$ on Politics-uk, while the performance of MMNMF-L-C increases when $\gamma$ is greater than 2 on Handwritten. In general, MMNMF-L-C and MMNMF-L-L perform well when $\gamma$ varies in $[0.4,4]$. In the following experiments, we set $\gamma=1$.

The proposed four algorithms are closely related to dimension reduction, we show their performances with various $r$ values in Figure 7 and Figure 8 , Generally, when $r$ is less than the number of clusters, the performances of the four algorithms become better and better with the increasing of $r$. After $r$ exceeds the number of clusters, the performances of MMNMF-L-L and MMNMF-R-L are generally stable with the increasing of $r$, while the performances of MMNMF-L$\mathrm{C}$ and MMNMF-R-C may increase at first and then decrease with the increasing of $r$. The four algorithms perform relatively well when $r$ equals to the number of clusters for each dataset. Thus for each dataset, the low rank $r$ is set as the number of clusters. 


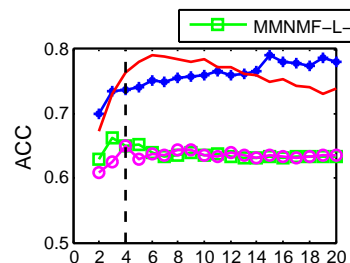

(a) Webkb

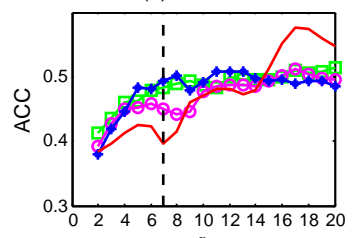

(d) Cora

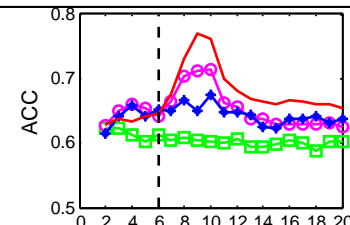

(b) 3 Sources

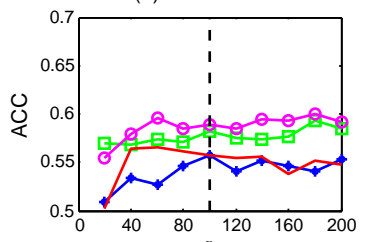

(e) ALO

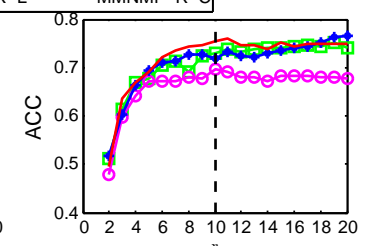

(c) Handwriten

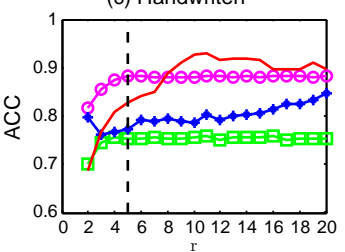

(f) Politics-uk

Figure 7: ACC of MMNMF on all datasets with various $r$. The abscissa of the dotted line equals to the number of clusters.

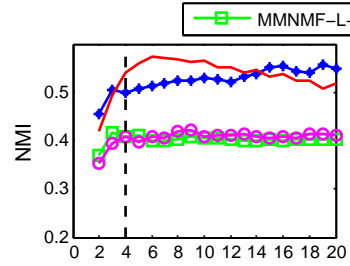

(a) Webkb

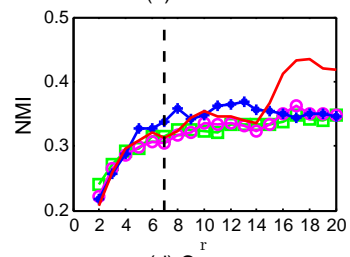

(d) Cora

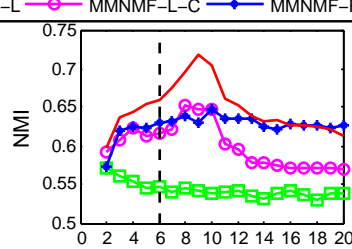

(b) 3 Sources

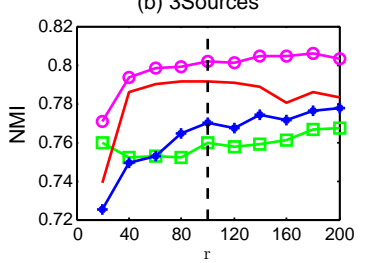

(e) ALOI

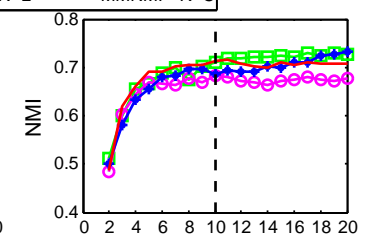

(c) Handwriten

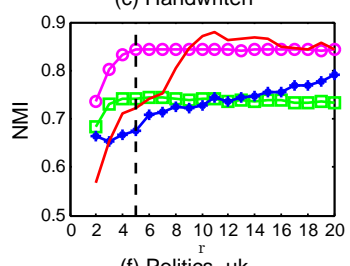

(f) Politics-uk

Figure 8: NMI of MMNMF on all datasets with various $r$. The abscissa of the dotted line equals to the number of clusters. 
We use KNN graph in SGNMF, MultiGNMF, MMNMF-L-L and MMNMFL-C. The number of nearest neighbors is set as 5 as Cai et al. (2011). The number of internal iterations $t_{i}$ is set as 10 for MultiNMF, MultiGNMF, MMNMF-L-C and MMNMF-R-C since the internal iteration converges in limited iterations. For other parameters in the baselines, we adopt the suggestions in their original papers. We run all the algorithms 20 times and report the average results.

\subsection{Results}

\subsubsection{Intrinsic structure discover}
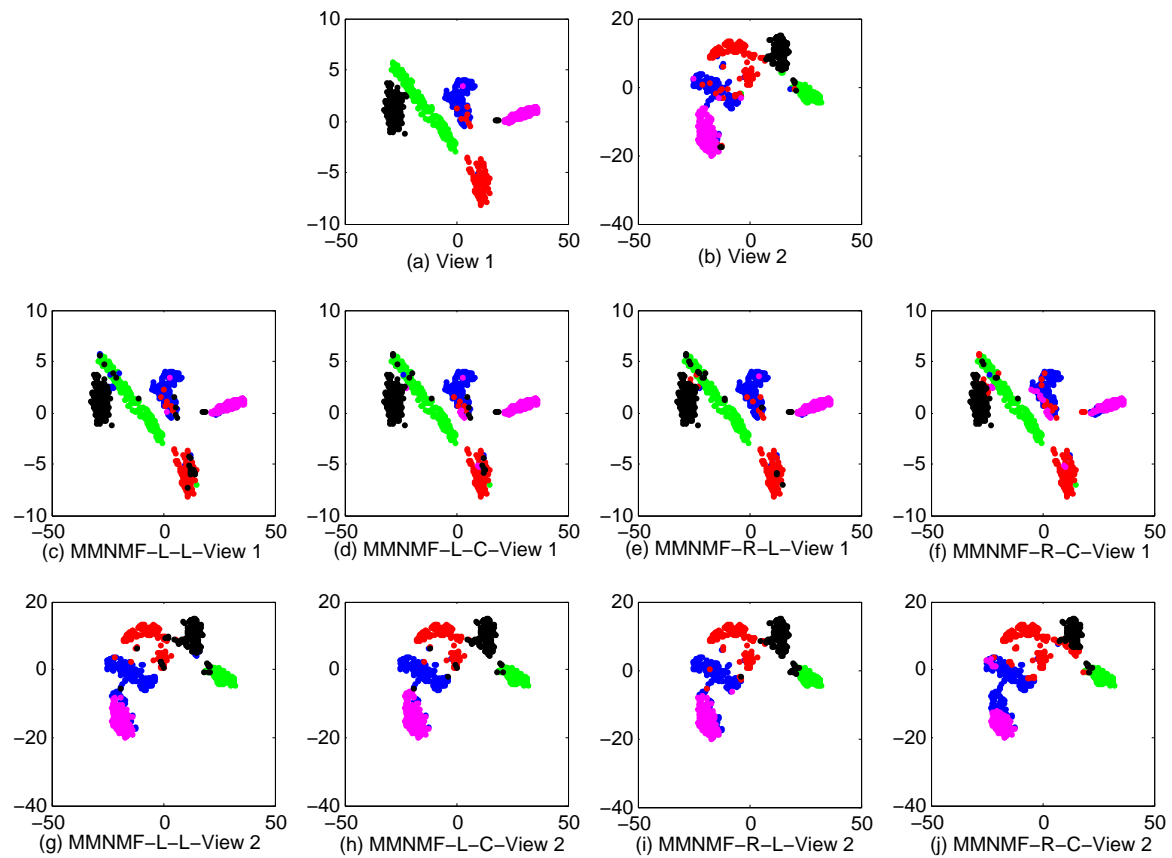

Figure 9: Handwritten visualization. (a) $\sim(b)$ : the structures of two views. $(\mathrm{c}) \sim(\mathrm{f})$ : the clustering results on view $1 .(\mathrm{g}) \sim(\mathrm{j})$ : the clustering results on view 2 .

The original motivation of manifold learning is to discover intrinsic structure. To verify this point, we provide the low-dimensional visualization of Handwritten with two views using t-Distributed Stochastic Neighbor Embedding (t-SNE) (Hinton (2008)). We visualize five clusters in Figure 9 where each color represents a cluster. The structures of the two views are shown in Figure 9. (a) 
and Figure 9.(b). Figure 9.(c) Figure 9.(f) are the clustering results of the proposed four algorithms on view 1, and Figure 9. (g) Figure 9.(j) are the clustering results of the proposed four algorithms on view 2 . It can be seen that the proposed four algorithms can discover the geometrical structure generally. The data points locating in the same manifold belong to the same cluster in most cases.

\subsubsection{Clustering results}

405

The clustering results in terms of ACC and NMI are reported in Table 3 and Table 4. respectively. In each column of the two tables, the best result is highlighted in boldface and marked by a star. Furthermore, we use paired t-test at 0.05 significance level and bold the performances without significant difference with the best performance. From the results, the following points can be observed.

- MMNMF-R-C performs better than the baselines in terms of ACC and NMI on all the datasets. MMNMF-L-L, MMNMF-L-C and MMNMF-R-L perform better than the baselines in most cases. Especially, the proposed algorithms may have $20 \%$ improvement on some datasets when compared with SGNMF.

- MMNMF-L-L, MMNMF-L-C, MMNMF-R-L and MMNMF-R-C alternatively perform the best in terms of NMI and ACC.

- In most cases, SGNMF performs better than SNMF and MultiGNMF performs better than MultiNMF in terms of ACC and NMI. R-L and MMNMF-R-C are the best four algorithms overall (considering both ACC and NMI metrics), which shows that multi-manifold regularization is effective for NMF based multi-view clustering. Algorithms with regularization on each single manifold, SGNMF and MultiGNMF, also improve upon their counterparts, SNMF and MultiNMF. However, the overall performances of SGNMF 
Table 3: Comparison of ACC on all datasets. The best average results are boldfaced and marked by a star. The performances without significant difference with the best performance are boldfaced (paired t-test at 0.05 significance level).

\begin{tabular}{|c|c|c|c|c|c|c|}
\hline & Webkb & 3Sources & Handwritten & Cora & ALOI & Politics-uk \\
\hline SNMF & $54.10 \pm 3.38$ & $50.21 \pm 2.92$ & $55.82 \pm 2.48$ & $33.09 \pm 1.05$ & $30.91 \pm 1.03$ & $57.43 \pm 1.58$ \\
ConcatNMF & $59.16 \pm 6.50$ & $55.38 \pm 9.91$ & $67.55 \pm 2.66$ & $37.17 \pm 1.95$ & $44.71 \pm 1.07$ & $68.62 \pm 7.98$ \\
SGNMF & $52.91 \pm 4.02$ & $53.83 \pm 1.19$ & $65.67 \pm 3.10$ & $37.24 \pm 2.25$ & $53.42 \pm 1.57$ & $59.57 \pm 1.47$ \\
ColNMF & $58.78 \pm 5.57$ & $58.73 \pm 7.51$ & $66.46 \pm 2.48$ & $36.95 \pm 2.41$ & $36.89 \pm 1.48$ & $68.75 \pm 6.39$ \\
MultiNMF & $60.27 \pm 2.63$ & $47.78 \pm 4.25$ & $68.37 \pm 2.82$ & $28.42 \pm 2.91$ & $50.39 \pm 1.70$ & $63.65 \pm 4.45$ \\
MultiGNMF & $66.90 \pm 6.11$ & $\mathbf{6 3 . 4 0} \pm \mathbf{7 . 6 9}$ & $\mathbf{7 3 . 9 9} \pm \mathbf{3 . 0 9}$ & $38.34 \pm 4.07$ & $54.89 \pm 1.88$ & $67.32 \pm 6.77$ \\
MMNMF-L-L & $66.62 \pm 7.24$ & $59.91 \pm 3.69$ & $\mathbf{7 2 . 7 4} \pm \mathbf{3 . 3 7}$ & $\mathbf{4 7 . 1 1} \pm \mathbf{5 . 1 3}$ & $57.37 \pm 1.56$ & $78.72 \pm 0.91$ \\
MMNMF-L-C & $63.94 \pm 6.30$ & $\mathbf{6 5 . 2 4} \pm \mathbf{7 . 1 8 *}$ & $69.50 \pm 6.78$ & $44.40 \pm 5.40$ & $\mathbf{5 8 . 6 4} \pm \mathbf{1 . 5 8 *}$ & $\mathbf{8 8 . 2 0} \pm \mathbf{0 . 6 3}$ \\
MMNMF-R-L & $\mathbf{7 4 . 1 8} \pm 3.71$ & $\mathbf{6 4 . 8 5} \pm \mathbf{4 . 5 4}$ & $\mathbf{7 0 . 9 6} \pm \mathbf{3 . 9 4}$ & $\mathbf{4 8 . 1 5} \pm \mathbf{4 . 8 4 *}$ & $54.78 \pm 1.63$ & $77.42 \pm 6.08$ \\
MMNMF-R-C & $\mathbf{7 7 . 1 0} \pm \mathbf{2 . 6 8 *}$ & $\mathbf{6 3 . 3 1} \pm \mathbf{4 . 1 2}$ & $\mathbf{7 4 . 2 0} \pm \mathbf{5 . 0 6 *}$ & $40.12 \pm 6.47$ & $55.59 \pm 1.99$ & $83.41 \pm 4.53$ \\
\hline
\end{tabular}

Table 4: Comparison of NMI on all datasets. The best average results are boldfaced and marked by a star. The performances without significant difference with the best performance are boldfaced (paired t-test at 0.05 significance level).

\begin{tabular}{|c|c|c|c|c|c|c|}
\hline & Webkb & 3Sources & Handwritten & Cora & ALOI & Politics-uk \\
\hline SNMF & $33.08 \pm 3.80$ & $50.29 \pm 1.87$ & $51.35 \pm 1.56$ & $15.88 \pm 1.02$ & $49.42 \pm 0.93$ & $51.78 \pm 1.81$ \\
ConcatNMF & $45.88 \pm 4.57$ & $56.01 \pm 6.30$ & $63.71 \pm 2.28$ & $18.27 \pm 1.33$ & $65.92 \pm 0.78$ & $73.93 \pm 3.96$ \\
SGNMF & $26.65 \pm 4.34$ & $51.33 \pm 0.51$ & $66.91 \pm 1.94$ & $19.78 \pm 2.02$ & $76.81 \pm 0.61$ & $44.43 \pm 1.51$ \\
ColNMF & $46.00 \pm 4.51$ & $58.15 \pm 4.88$ & $61.62 \pm 2.25$ & $18.52 \pm 1.82$ & $58.99 \pm 0.81$ & $74.28 \pm 1.69$ \\
MultiNMF & $46.81 \pm 3.91$ & $48.14 \pm 3.03$ & $64.68 \pm 2.34$ & $8.73 \pm 2.30$ & $71.73 \pm 0.86$ & $72.59 \pm 4.29$ \\
MultiGNMF & $48.22 \pm 3.16$ & $60.92 \pm 4.68$ & $\mathbf{6 9 . 6 6} \pm \mathbf{2 . 1 9}$ & $22.34 \pm 3.29$ & $75.68 \pm 0.77$ & $73.14 \pm 1.74$ \\
MMNMF-L-L & $41.17 \pm 7.65$ & $53.98 \pm 3.48$ & $\mathbf{7 1 . 1 9} \pm \mathbf{2 . 1 4} *$ & $31.09 \pm 3.61$ & $75.61 \pm 0.65$ & $76.90 \pm 0.86$ \\
MMNMF-L-C & $40.82 \pm 6.04$ & $62.15 \pm 5.65$ & $68.12 \pm 4.91$ & $30.29 \pm 2.91$ & $\mathbf{7 9 . 9 2} \pm \mathbf{0 . 5 2}$ & $\mathbf{8 4 . 3 7} \pm \mathbf{0 . 7 4 *}$ \\
MMNMF-R-L & $51.04 \pm 5.02$ & $63.27 \pm 2.54$ & $\mathbf{6 9 . 7 7} \pm \mathbf{4 . 2 8}$ & $\mathbf{3 3 . 2 4} \pm \mathbf{4 . 6 7 *}$ & $76.79 \pm 0.78$ & $67.94 \pm 4.00$ \\
MMNMF-R-C & $\mathbf{5 4 . 3 4} \pm \mathbf{3 . 3 9 *}$ & $\mathbf{6 5 . 6 9} \pm \mathbf{2 . 3 4 *}$ & $\mathbf{7 0 . 5 2} \pm \mathbf{4 . 3 9}$ & $\mathbf{3 2 . 0 1} \pm \mathbf{3 . 2 4}$ & $78.92 \pm 0.79$ & $74.99 \pm 4.38$ \\
\hline
\end{tabular}

and MultiGNMF are not as good as those of MMNMF-L-L, MMNMF-L-C, MMNMF-R-L and MMNMF-R-C. Among all the four instances, the overall performance of MMNMF-R-C is the best: it performs the best or its performance does not have significant difference with the best performance in most cases.

\subsection{Algorithm convergence}

The update rules for minimizing the objective functions of MMNMF-L-L, MMNMF-L-C, MMNMF-R-L and MMNMF-R-C are essentially iterative. We investigate the convergence of the rules on all the datasets. Figure 10 shows the convergence curve of the objective functions (in log values). As we can see, on all the datasets, the objective values fall fast within 10 iterations. The algorithms will converge within 500 iterations except for MMNMF-L-L on Politics-uk. 


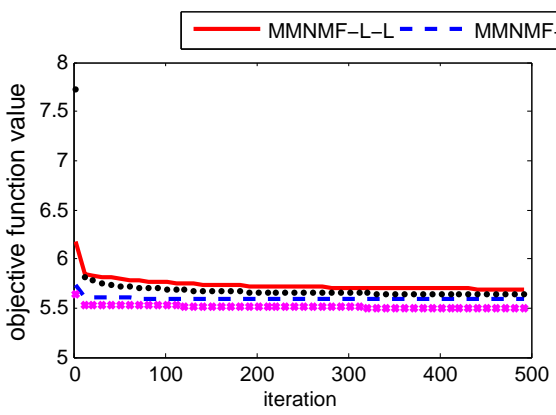

(a) Webkb

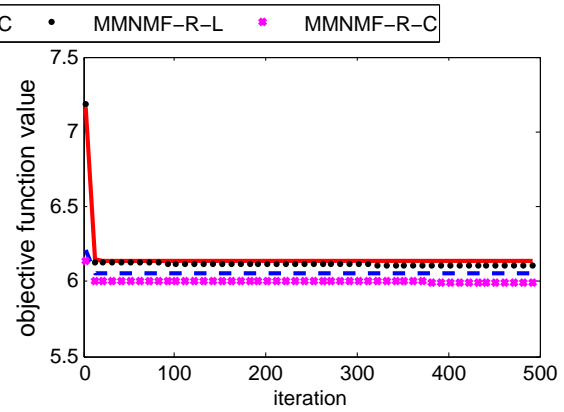

(b) 3Sources

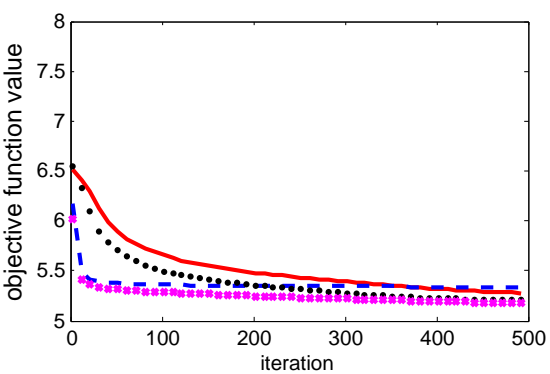

(c) Handwriten

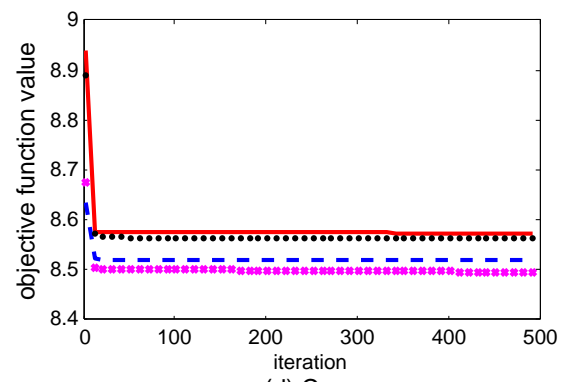

(d) Cora

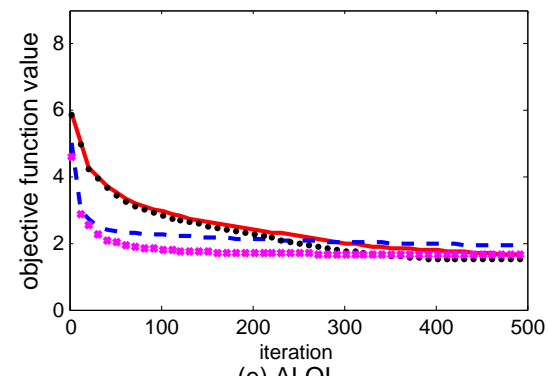

(e) ALOI

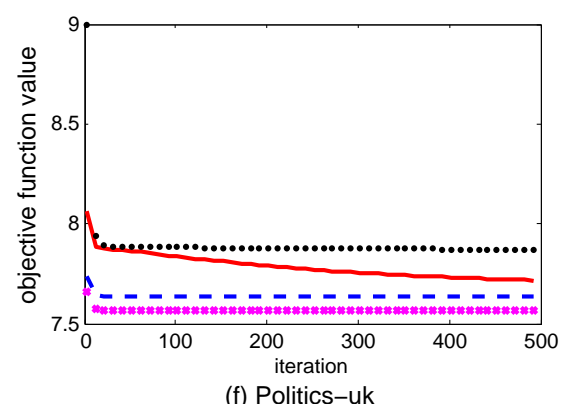

Figure 10: Convergence study on all datasets. The horizontal axis shows the number of iterations and the vertical axis shows the log values of objective functions. 
Table 5: Summary of the totally computational complexity.

\begin{tabular}{|c|c|}
\hline Algorithm & Complexity \\
\hline SNMF & $O\left(t_{o} n n_{v} m r\right)$ \\
\hline ConcatNMF & $O\left(t_{o} n r\left(\sum_{v} m^{(v)}\right)\right)$ \\
\hline SGNMF & $O\left(t_{o} n n_{v} m r+t_{o} n^{2} n_{v} r+n^{2} m n_{v}\right)$ \\
\hline ColNMF & $O\left(t_{o} n n_{v} m r\right)$ \\
\hline MultiNMF & $O\left(t_{o} t_{i} n n_{v} m r\right)$ \\
\hline MultiGNMF & $O\left(t_{o} t_{i} n^{2} n_{v} r+t_{o} t_{i} n n_{v} m r+n^{2} m n_{v}\right)$ \\
\hline MMNMF-L-L & $O\left(t_{o} n_{v}^{2} n^{2} r+t_{o} n n_{v} m r+n^{2} m n_{v}\right)$ \\
\hline MMNMF-L-C & $O\left(t_{o} n^{2} n_{v} r+t_{o} t_{i} n n_{v} m r+n^{2} m n_{v}\right)$ \\
\hline MMNMF-R-L & $O\left(t_{o} n_{v}^{2} n^{2} r+t_{o} n n_{v} m r+n n^{\prime} m n_{v}\right)$ \\
\hline MMNMF-R-C & $O\left(t_{o} n^{2} r+t_{o} t_{i} n n_{v} m r+n n^{\prime} m n_{v}\right)$ \\
\hline
\end{tabular}

\subsection{Running time}

In Figure 11, we compare the total computational time of MMNMF with

440 that of the baselines. When clustering data with multiple views, the total computational complexities of all the algorithms are shown in Table 5, where $m=$ $\max _{v} m^{(v)}, n_{I}^{i}=\left|I\left(x_{i}\right)\right|$ is the total number of $x_{i}$ 's neighbors, $n_{I}=\max _{i} n_{I}^{i}$, $n^{\prime}=\max \left\{n, n_{I}^{3}\right\}, t_{o}$ is the number of iterations and $t_{i}$ is the number of internal iterations. Since MultiGNMF finds a consensus coefficient matrix in the process of iteration, we implement it by using the method in MultiNMF (Liu et al. (2013)). We set $t_{o}=50$ for all methods. Also, we set $t_{i}=10$ for MultiGNMF, MMNMF-L-C and MMNMF-R-C since the internal iteration converges in limited iterations. For single view methods, SNMF and SGNMF, we report the running time of all views. Generally, manifold based methods are more time-consuming than the corresponding methods without manifold regularization, and manifold based multi-view methods require more running time than others. Specifically, MultiGNMF needs the most computational time due to the high complexity. Besides MultiGNMF, MMNMF is more time-consuming than the other algorithms. However, considering the improvement on clustering performance, the increase of computational time is worthwhile.

The computational time of MMNMF mainly contains the time of updating matrices and determining parameters. In Figure 12 , we separately show the computational time of updating matrices and determining parameters. We find 

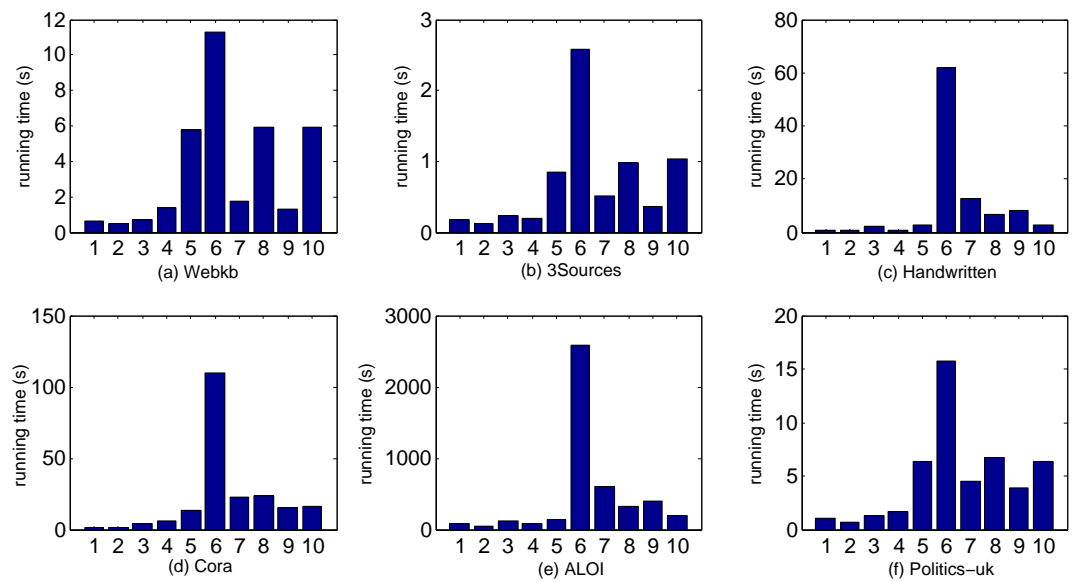

Figure 11: Comparison of running time on all datasets. The number $1 \sim 10$ in the horizontal axis of each figure separately represent SNMF, ConcatNMF, SGNMF, ColNMF, MultiNMF, MultiGNMF, MMNMF-L-L, MMNMF-L-C, MMNMF-R-L and MMNMF-R-C. The number of iterations is set as 50 for all methods. For MultiGNMF, MMNMF-L-C and MMNMF-R-C, the number of internal iterations is set as 10 .

that updating matrices is more time-consuming in MMNMF-L-C and MMNMF$\mathrm{R}-\mathrm{C}$ on all the datasets because of the high complexity. For MMNMF-L$\mathrm{L}$ and MMNMF-R-L, updating matrices is more time-consuming on Webkb and 3Sources while less time-consuming on the others. In MMNMF-L-L and MMNMF-R-L, the computational complexity of updating matrices and determining parameters separately are $O\left(t_{o} n^{2} n_{v} r+t_{o} n n_{v} m r\right)$ and $O\left(t_{o} n^{2} n_{v}^{2} r\right)$. Then, updating matrices maybe more time-consuming if the dimension is larger than the number of data points, and vice versa.

\section{Conclusion}

In this paper, we propose a multi-manifold regularized NMF framework for multi-view clustering, which preserves the locally geometrical structure of multiple data spaces. The framework provides flexibility on finding the consensus coefficient matrix and the consensus manifold. In particular, we have realized four instances of the framework with different strategies to find the consen- 


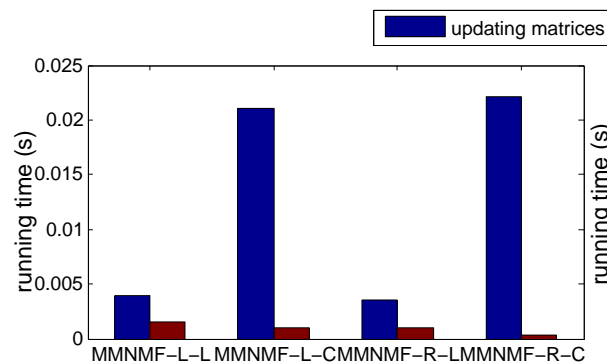

(a) Webkb

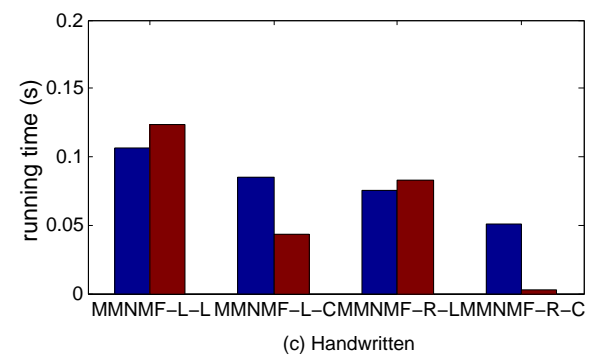

(c) Handwritten

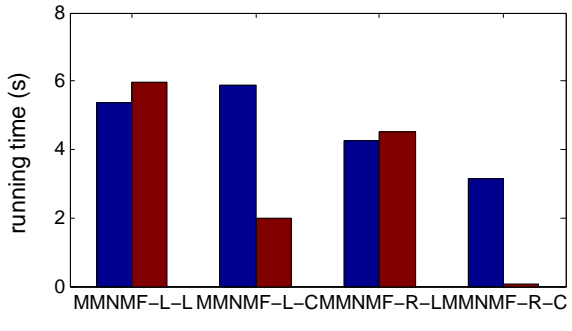

(e) ALOI

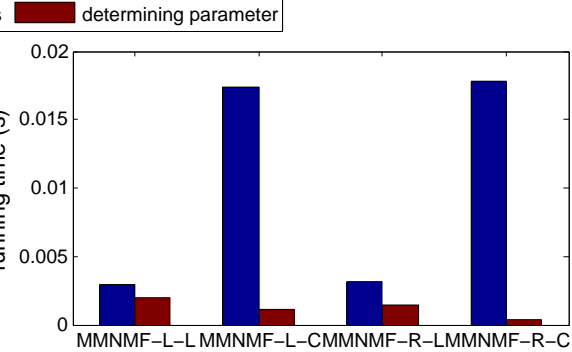

(b) 3 Sources
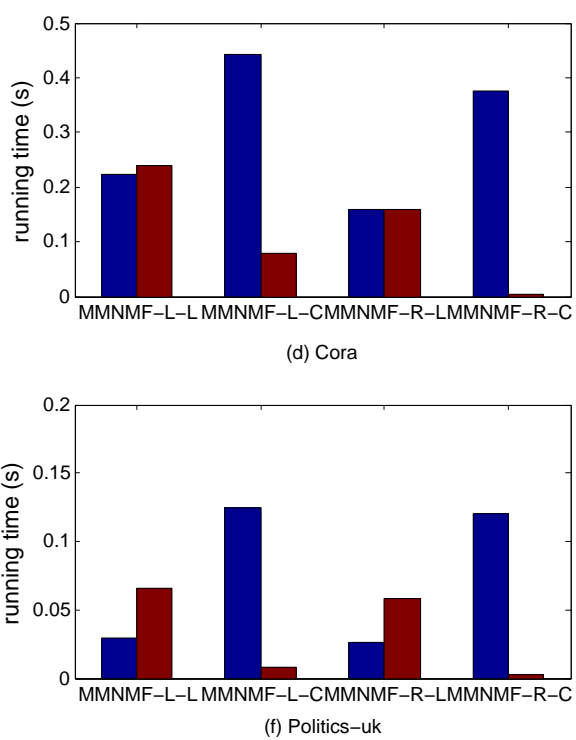

Figure 12: Running time of updating matrices and determining parameters. For MultiGNMF, MMNMF-L-C and MMNMF-R-C, the number of internal iterations is set as 10 . 
sus coefficient matrix and consensus manifold. Experimental results on several benchmark datasets have demonstrated the superiorities of the proposed algorithms.

\section{Acknowledgments}

This work was supported by National Science Foundation of China (No. 61272374,61428202) and National High Technology Research and Development Program (863 Program) of China (No. 2015AA015403).

480

Aggarwal, C. C., \& Reddy, C. K. (2013). Data clustering: algorithms and applications. Boca Raton, Florida: CRC Press.

Akata, Z., Thurau, C., \& Bauckhage, C. (2011). Non-negative matrix factorization in multimodality data for segmentation and label prediction. In 16th Computer Vision Winter Workshop (CVWW) (pp. 1-8).

Aldous, D., \& Fill, J. (2002). Reversible Markov chains and random walk$s$ on graphs. Online version available at http://www.stat.berkeley.edu/ aldous/RWG/book.html.

Beck, A., \& Teboulle, M. (2003). Mirror descent and nonlinear projected sub490 gradient methods for convex optimization. OPER RES LETT., 31, 167-175.

Bickel, S., \& Scheffer, T. (2004). Multi-view clustering. In IEEE International Conference on Data Mining (ICDM) (pp. 19-26). volume 4.

Boyd, S., \& Vandenberghe, L. (2004). Convex optimization. Cambridge, England: Cambridge university press.

${ }_{495}$ Cai, D., He, X., Han, J., \& Huang, T. S. (2011). Graph regularized nonnegative matrix factorization for data representation. IEEE Trans. Pattern Anal. Mach. Intell., 33, 1548-1560. 
Cai, X., Nie, F., \& Huang, H. (2013). Multi-view k-means clustering on big data. In Proceedings of the 23rd International joint conference on Artificial Intelligence (IJCAI) (pp. 2598-2604).

Cheng, H., Liu, Z., \& Yang, J. (2009). Sparsity induced similarity measure for label propagation. In IEEE 12th International Conference on Computer Vision (ICCV) (pp. 317-324).

Chung, F. R. (1997). Spectral graph theory volume 92. Providence, RI: American Mathematical Soc.

Ding, C. H., He, X., \& Simon, H. D. (2005). On the equivalence of nonnegative matrix factorization and spectral clustering. In proceedings of the 5th SIAM International Conference on Data Mining (SDM) (pp. 606-610).

Geng, B., Tao, D., Xu, C., Yang, Y., \& Hua, X.-S. (2012). Ensemble manifold regularization. IEEE Trans. Pattern Anal. Mach. Intell., 34, 1227-1233.

Greene, D., \& Cunningham, P. (2013). Producing a unified graph representation from multiple social network views. In Proceedings of the 5th Annual ACM Web Science Conference (pp. 118-121).

Hinton, G. E. (2008). Visualizing high-dimensional data using t-sne. Vigiliae Christianae, 9, 2579-2605.

Kuang, D., Park, H., \& Ding, C. H. (2012). Symmetric nonnegative matrix factorization for graph clustering. In Proceedings of the 12th SIAM International Conference on Data Mining (SDM) (pp. 106-117).

Kumar, A., \& Daumé, H. (2011). A co-training approach for multi-view spectral clustering. In Proceedings of the 28th International Conference on Machine Learning (ICML) (pp. 393-400).

Kumar, A., Rai, P., \& Daume, H. (2011). Co-regularized multi-view spectral clustering. In Advances in Neural Information Processing Systems (NIPS) (pp. 1413-1421). 
Lee, D. D., \& Seung, H. S. (1999). Learning the parts of objects by non-negative matrix factorization. Nature., 401, 788-791.

Lee, D. D., \& Seung, H. S. (2001). Algorithms for non-negative matrix factorization. In Advances in neural information processing systems (NIPS) (pp. $556-562)$.

Li, P., Bu, J., \& Cai, D. (2013). Multi-manifold concept factorization for data clustering. Int J Software Informatics., 7, 407-418.

Li, S.-Y., Jiang, Y., \& Zhou, Z.-H. (2014). Partial multi-view clustering. In Twenty-Eighth AAAI Conference on Artificial Intelligence (pp. 1968-1974).

Liu, J., Wang, C., Gao, J., \& Han, J. (2013). Multi-view clustering via joint nonnegative matrix factorization. In Proceedings of the 13th SIAM International Conference on Data Mining (SDM) (pp. 252-260).

Liu, X., Lu, H., \& Gu, H. (2005). Group sparse non-negative matrix factorization for multi-manifold learning. Intelligence., 27, 1945-1959.

Roweis, S. T., \& Saul, L. K. (2000). Nonlinear dimensionality reduction by locally linear embedding. Science., 290, 2323-2326.

Shen, B., \& Si, L. (2010). Non-negative matrix factorization clustering on multiple manifolds. In Proceedings of the 24th AAAI Conference on Artificial Intelligence (AAAI).

Singh, A. P., \& Gordon, G. J. (2008). Relational learning via collective matrix factorization. In Proceedings of the 14th ACM SIGKDD International conference on Knowledge discovery and data mining (SIGKDD) (pp. 650-658).

Tian, Z., \& Kuang, R. (2012). Global linear neighborhoods for efficient label propagation. In Proceedings of the 12th SIAM International Conference on Data Mining (SDM) (pp. 863-872).

${ }_{550}$ Von Luxburg, U. (2007). A tutorial on spectral clustering. Statistics and computing, 17, 395-416. 
Wang, F., \& Zhang, C. (2008). Label propagation through linear neighborhoods. IEEE Trans. Knowl. Data Eng., 20, 55-67.

Wang, Y., Jiang, Y., Wu, Y., \& Zhou, Z. H. (2011). Local and structural consistency for multi-manifold clustering. In International Joint Conference on Artificial Intelligence (pp. 1559-1564).

Xu, W., Liu, X., \& Gong, Y. (2003). Document clustering based on nonnegative matrix factorization. In Proceedings of the 26th Annual International ACM SIGIR conference on Research and development in informaion retrieval (SIGIR) (pp. 267-273).

Yan, S., \& Wang, H. (2009). Semi-supervised learning by sparse representation. In Proceedings of the 9th SIAM International Conference on Data Mining (SDM) (pp. 792-801).

Yang, N., Sang, Y., He, R., \& Wang, X. (2010). Label propagation algorithm based on non-negative sparse representation. In Life System Modeling and Intelligent Computing (pp. 348-357).

Zhang, X., Zhao, L., Zong, L., Liu, X., \& Yu, H. (2014). Multi-view clustering via multi-manifold regularized nonnegative matrix factorization. In IEEE International Conference on Data Mining (ICDM) (pp. 1103-1108).

Zhang, X., Zong, L., Liu, X., \& Yu, H. (2015). Constrained nmf-based multiview clustering on unmapped data. In Twenty-Ninth AAAI Conference on Artificial Intelligence (pp. 3174-3180).

Zhou, D., \& Burges, C. J. (2007). Spectral clustering and transductive learning with multiple views. In Proceedings of the 24th International conference on Machine learning (ICML) (pp. 1159-1166). 